\title{
Manganese-containing Prussian blue nanoparticles for imaging of pediatric brain tumors
}

\author{
This article was published in the following Dove Press journal: \\ International Journal of Nanomedicine \\ 23 May 2014 \\ Number of times this article has been viewed
}

\section{Matthieu F Dumont \\ Sridevi Yadavilli ${ }^{2}$ \\ Raymond W Sze ${ }^{1,4}$ \\ Javad Nazarian ${ }^{2,3}$ \\ Rohan Fernandes $1,4,5$}

'Sheikh Zayed Institute for Pediatric Surgical Innovation, ${ }^{2}$ Center for Genetic Medicine Research, Children's National Medical Center, Washington, DC, USA; ${ }^{3}$ Department of Integrative Systems Biology, ${ }^{4}$ Department of Radiology, ${ }^{5}$ Department of Pediatrics, George Washington University, Washington, DC, USA
Correspondence: Rohan Fernandes Sheikh Zayed Institute for Pediatric Surgical Innovation, Children's National Medical Center, I I I Michigan Ave NW, Washington, DC 200I0, USA

$\mathrm{Tel}+\mathrm{I} 2024765290$

Email RFernand@childensnational.org

\begin{abstract}
Pediatric brain tumors (PBTs) are a leading cause of death in children. For an improved prognosis in patients with PBTs, there is a critical need to develop molecularlyspecific imaging agents to monitor disease progression and response to treatment. In this paper, we describe manganese-containing Prussian blue nanoparticles as agents for molecular magnetic resonance imaging (MRI) and fluorescence-based imaging of PBTs. Our core-shell nanoparticles consist of a core lattice structure that incorporates and retains paramagnetic $\mathrm{Mn}^{2+}$ ions, and generates MRI contrast (both negative and positive). The biofunctionalized shell is comprised of fluorescent avidin, which serves the dual purpose of enabling fluorescence imaging and functioning as a platform for the attachment of biotinylated ligands that target PBTs. The surfaces of our nanoparticles are modified with biotinylated antibodies targeting neuron-glial antigen 2 or biotinylated transferrin. Both neuron-glial antigen 2 and the transferrin receptor are protein markers overexpressed in PBTs. We describe the synthesis, biofunctionalization, and characterization of these multimodal nanoparticles. Further, we demonstrate the MRI and fluorescence imaging capabilities of manganese-containing Prussian blue nanoparticles in vitro. Finally, we demonstrate the potential of these nanoparticles as PBT imaging agents by measuring their organ and brain biodistribution in an orthotopic mouse model of PBTs using ex vivo fluorescence imaging.
\end{abstract}

Keywords: Prussian blue, nanoparticles, imaging, pediatric brain tumors, fluorescence, magnetic resonance imaging, manganese, multimodal

\section{Introduction}

Pediatric brain tumors (PBTs) are the most prevalent type of solid tumor in children and one of the leading causes of cancer-related death in the pediatric age group. ${ }^{1,2}$ The prognosis for children with aggressive PBTs such as glioblastoma multiforme, medulloblastoma, and diffuse intrinsic pontine glioma is poor, with virtually no long-term survivors. $^{3-5}$ The reasons for the poor success in improving patient survival can be attributed to a historical lack of knowledge of the underlying biology of aggressive PBTs, and the lack of molecularly-specific imaging modalities to monitor disease progression and evaluate the response to treatment. ${ }^{6}$

Over the past decade, technological progress made in the field of molecular biology coupled with increased access to tissue samples from PBT patients (obtained via biopsy and/or autopsy) and the development of relevant animal models have improved our understanding of the underlying molecular biology of PBTs. ${ }^{3,6}$ However, thus far, there are no clinically approved, molecularly-specific imaging agents for PBTs, which can be primarily attributed to the challenges of working in the brain and central nervous system, including crossing the blood-brain barrier and penetrating the brain parenchyma. In this paper, we describe a manganese-containing Prussian blue 
(MnPB) nanoparticle for multimodal molecular magnetic resonance imaging (MRI) and fluorescence-based imaging of PBTs.

Our rationale for pursuing nanoparticles is that they offer unique advantages that can be harnessed for molecular imaging of PBTs, ${ }^{7}$ including: small sizes $(\sim 10-200 \mathrm{~nm})$ that enable the nanoparticles to easily penetrate body barriers and extravasate across the leaky tumor vasculature of PBTs (via the enhanced permeability and retention effect, on account of the abnormal molecular and fluid transport dynamics of these tumors); ${ }^{8-10}$ high surface area-to-volume ratios that enable attachment of PBT-targeting ligands with high density; and the ability to be visualized via imaging modalities such as MRI or fluorescence. ${ }^{11-14}$ There are numerous examples in the literature of nanoparticles being used for the imaging and treatment of a variety of cancers, including gold nanoparticles/nanorods/nanoshells, iron oxide nanoparticles, carbon nanotubes, dendrimers, polymer-based nanoparticles, and quantum dots. ${ }^{15-18}$ While a few of these nanoparticles are currently undergoing clinical evaluation, ${ }^{7,19-23}$ their use for molecular imaging of PBTs has been limited, thereby representing an opportunity for the field of nanomedicine.

To generate nanoparticles suitable for imaging PBTs, we have synthesized MnPB nanoparticles with a core-shell design (Figure 1A). The Prussian blue nanoparticle core (iron [III] hexacyanoferrate [II]) has a permeable lattice structure and the ability to incorporate and retain metal ions for charge balance (Figure 1B). On account of this metal-binding ability, Prussian blue, a material approved by the US Food and Drug Administration and sold as Radiogardase $^{\mathrm{TM}}$ (Heyltex Corporation, Katy, TX, USA), ${ }^{24}$ has been used by first responders in the event of a radiological emergency to sequester and promptly eliminate radioactive isotopes of cesium and thallium from the body. ${ }^{25,26}$ For the purpose of MRI, we can harness this metal-binding ability of Prussian blue to sequester paramagnetic $\mathrm{Mn}^{2+}$ ions within its lattice, thereby generating MRI contrast. We use manganese ions in the nanoparticles to mitigate safety concerns associated with gadolinium ions $\left(\mathrm{Gd}^{3+}\right.$, typically used in commercial contrast agents) that have been linked to nephrogenic systemic fibrosis in patients with impaired renal function. ${ }^{27-30}$

The Prussian blue nanoparticle core is coated with fluorescently labeled avidin as previously described. ${ }^{31}$ The avidin coating biofunctionalizes the nanoparticle and serves as a platform for attaching biotinylated ligands for tumor targeting. ${ }^{32,33}$ The fluorescent avidin enables fluorescence

\section{A Manganese-containing Prussian blue (MRI contrast agent)}
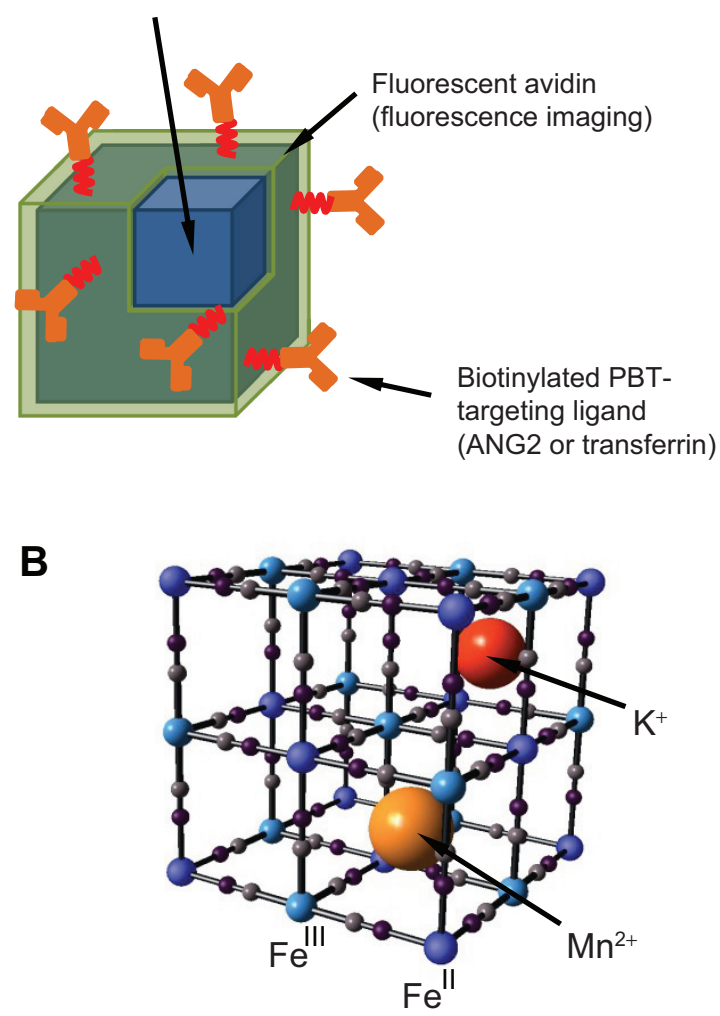

Figure I Manganese-containing Prussian blue nanoparticles for imaging of PBTs. Notes: (A) Schematic representation of manganese-containing Prussian blue nanoparticles comprised of an inorganic core that provides MRI contrast and a biofunctionalized shell consisting of fluorescently-labeled avidin and biotinylated PBT-targeting ligands. (B) Schematic representation of the Prussian blue lattice (ball and stick) containing interstitial $\mathrm{Mn}^{2+}$ and $\mathrm{K}^{+}$ions.

Abbreviations: ANG2, anti-neuron-glial antigen 2; MRI, magnetic resonance imaging; PBT, pediatric brain tumor.

imaging and detection of our nanoparticles. Our reason for selecting MRI and fluorescence as the molecular imaging modalities is on account of their complementary features. MRI offers superior spatial resolution and limitless penetration depths, while fluorescence imaging can be used to image phenomena with up to picomolar sensitivity. ${ }^{34}$ Further, MRI is the standard imaging modality for diagnosis and follow-up of PBTs, ${ }^{35}$ while fluorescence can be used to sensitively outline tumor margins, which is important when planning surgical resection of PBTs. ${ }^{36}$ To confer PBT molecular targeting capabilities, we utilize two different biotinylated ligands attached to the avidin-coated nanoparticles: a biotinylated antibody targeting neuron-glial antigen 2 , which is a cell surface chondroitin sulfate proteoglycan that has been observed to be overexpressed in numerous PBTs, including glioblastoma multiforme and diffuse intrinsic pontine gliomas; ${ }^{37-39}$ and biotinylated transferrin, a glycoprotein that has been shown to specifically target a variety of tumors, 
including PBTs, and to assist various cargo in crossing the blood-brain barrier. ${ }^{40-42}$

Here we describe the synthesis, biofunctionalization, and characterization of the MnPB nanoparticles. We also evaluate the suitability of these nanoparticles for MRI and fluorescence-based imaging of PBTs in vitro using cells derived from brain tumors of a genetically engineered mouse model of PBT. Finally, we evaluate the imaging capabilities of our nanoparticles by studying their organ and brain biodistribution in an orthotopic mouse model of PBT using ex vivo fluorescence imaging.

\section{Materials and methods Materials}

All synthetic procedures were conducted using ultrapure water obtained from a Milli-Q system (Millipore Corporation, Billerica, MA, USA) with resistivity $\geq 18 \mathrm{M} \Omega \mathrm{cm}$. All chemicals and reagents were purchased from Fisher-Acros (Thermo Fisher Scientific, Waltham, MA, USA) or SigmaAldrich (St Louis, MO, USA), and used as supplied.

\section{Synthesis of MnPB nanoparticles}

Synthesis of the MnPB nanoparticles was carried out at ambient temperature by addition of a solution containing $0.0049 \mathrm{~g}$ $\mathrm{FeCl}_{2} \cdot 4 \mathrm{H}_{2} \mathrm{O}\left(2.5 \times 10^{-2} \mathrm{mmol}\right)$ in $5 \mathrm{~mL}$ of Milli-Q water to a solution containing $0.0049 \mathrm{~g} \mathrm{MnCl}_{2} \cdot 4 \mathrm{H}_{2} \mathrm{O}\left(2.5 \times 10^{-2} \mathrm{mmol}\right)$ in $10 \mathrm{~mL}$ of Milli-Q water with vigorous stirring. This step was followed by immediate addition of a solution containing $0.0092 \mathrm{~g}$ of $_{3} \mathrm{Fe}(\mathrm{CN})_{6}\left(2.8 \times 10^{-2} \mathrm{mmol}\right)$ in $5 \mathrm{~mL}$ of Milli-Q water. After stirring for a further 15 minutes, the resulting nanoparticle precipitate was collected by centrifuging, rinsed with copious amounts of Milli-Q water, and redispersed in Milli-Q water. Energy-dispersive X-ray spectroscopy of the blue powder obtained using the synthesis scheme yielded the formula: $\mathrm{K}_{0.6} \mathrm{Mn}_{0.7} \mathrm{Fe}^{\mathrm{III}}{ }_{4}\left[\mathrm{Fe}^{\mathrm{II}}(\mathrm{CN})_{6}\right]_{3.5} \cdot 3 \mathrm{H}_{2} \mathrm{O}$.

\section{Physical characterization of MnPB nanoparticles}

Transmission electron microscopy (TEM) of the MnPB nanoparticles was performed using a JEM-2100 FEG highresolution instrument (JEOL, Ltd, Tokyo, Japan) at $200 \mathrm{kV}$. Energy-dispersive X-ray spectroscopy was performed using an Inca 250 (Oxford Instruments, Abingdon, UK) coupled to the high-resolution TEM. Three scans of different parts of the MnPB sample were performed and averaged to obtain the relative percentages of iron, potassium, and manganese (Supplementary materials). X-ray diffraction patterns of the
MnPB nanoparticles were measured on a D8 Advance powder diffractometer using $\mathrm{CuK} \alpha$ radiation, and Topas software (Bruker AXS GmbH, Karlsruhe, Germany) was utilized for peak fitting via a fundamental parameters approach. Fourier transform infrared (FTIR) spectra of the MnPB nanoparticles were recorded on an FTIR spectrometer (Thermo Nicolet Nexus 670, Thermo Fisher Scientific) using MnPB nanoparticle powders pressed against a ZnSe-attenuated total reflectance anvil.

\section{Measurements of magnetic resonance relaxation times and data analysis of MnPB nanoparticles}

All magnetic resonance measurements were performed in a horizontal $3 \mathrm{~T}$ clinical magnet (GE Healthcare, Little Chalfont, UK). The sample-containing phantom was placed adjacent to a block containing $2 \%$ agar $\left(150 \mathrm{~cm}^{3}\right)$ and secured at the center of an eight-channel HD brain coil (GE Healthcare). The MRI scanner was interfaced with the built-in GE Healthcare software. The magnetic resonance data were analyzed using ImageJ (National Institutes of Health, Bethesda, MD, USA). For each sample, the signal intensity of the acquired images was averaged over the area of the sample. The magnetic resonance images for MnPB nanoparticles were acquired using clinical GE Healthcare sequences (T1 Cube, echo train 24; repetition time $600 \mathrm{msec}$; echo time $11.0 \mathrm{msec}$ for T1-weighted, initial flip angle $120^{\circ}$ and minimum flip angle $25^{\circ}$; and $\mathrm{T} 2 \mathrm{Cube}$, echo train 100 ; repetition time 2,500 msec; echo time $79.6 \mathrm{msec}$ for T2-weighted). The concentrations of Prussian blue and MnPB measured were $8.500,4.250,2.120,1.062,0.531,0.265,0.132,0.066$, $0.033,0.016,0.008$, and $0.004 \mathrm{mg} / \mathrm{mL}$.

\section{Coating MnPB nanoparticles with fluorescent avidin}

A $0.9 \mathrm{~mL}$ suspension of MnPB in Milli-Q water $(180 \mu \mathrm{g} / \mathrm{mL})$ and $0.1 \mathrm{~mL}$ of filtered Alexa Fluor 488-labeled avidin solution (A488, $1 \mathrm{mg} / \mathrm{mL}$; Life Technologies, Carlsbad, CA, USA) or Avidin-Texas Red (ATxRd, $1 \mathrm{mg} / \mathrm{mL}$; Life Technologies) were mixed by vortexing. The mixture was protected from light and contacted on an orbital shaker for at least 3 hours at $4^{\circ} \mathrm{C}$. After contacting MnPB with fluorescent avidin, the unbound avidin was rinsed by centrifugation for 5 minutes at $20,000 \times g$ three times. In between each centrifugation, the supernatant was discarded and the particles (ie, MnPBA488 or MnPB-ATxRd) resuspended by brief sonication in $0.5 \mathrm{~mL}$ of Milli-Q water. After the last centrifugation, the particles were resuspended in Milli-Q water. 


\section{Attaching biotinylated ligands onto Avidin-coated MnPB}

A $0.9 \mathrm{~mL}$ dispersion of avidin-coated MnPB nanoparticles in Milli-Q water $(180 \mu \mathrm{g} / \mathrm{mL})$ and $0.1 \mathrm{~mL}$ of filtered, biotinylated anti-neuron-glial antigen 2 (ANG2; Millipore Corporation, Billerica, MA, USA), biotinylated eotaxin-3 antibody (AbC; PeproTech Inc) or biotinylated human transferrin (Rockland Immunochemicals, Inc., Gilbertsville, PA, USA) were mixed. The mixture was protected from light and contacted on an orbital shaker for at least 3 hours at $4^{\circ} \mathrm{C}$. After reaction, the unbound biotinylated ligands were removed by rinsing and the resulting biofunctionalized particles (ie, MnPB-A488-ANG2, MnPB-A488-AbC, or MnPB-ATxRdtransferrin) were resuspended in Milli-Q water as described previously. The names and components of the nanoparticles used in this study are summarized in Table S1.

\section{Nanoparticle size, charge, and temporal stability}

The sizes and zeta potentials of the suspensions of MnPB, avidin-coated MnPB, and biotinylated ligand-modified avidin-coated MnPB nanoparticles $(10 \mu \mathrm{g} / \mathrm{mL})$ were determined using a Zetasizer Nano ZS (Malvern Instruments, Malvern, UK). The temporal stability of the nanoparticles was determined in Milli-Q water and Dulbecco's Modified Eagle's Medium (Life Technologies).

\section{Cells used for study}

Adherent mouse brainstem glioma (BSG) D10 cells and nonadherent mouse neurospheres were derived from a genetically engineered mouse model of BSG as described previously. ${ }^{43}$ BSG D10 cells were cultured in Dulbecco's Modified Eagle's Medium containing high glucose supplemented with $10 \%$ fetal bovine serum (Life Technologies) and 100 units $/ \mathrm{mL}$ of penicillin plus $100 \mu \mathrm{g} / \mathrm{mL}$ of streptomycin (Life Technologies). The cultures were maintained at $5 \% \mathrm{CO}_{2}$ and $37^{\circ} \mathrm{C}$. The mouse neurospheres were cultured in mouse Neurocult medium (Stem Cell Technologies, Vancouver, BC, Canada) supplemented with 10\% mouse cell proliferation supplement (Stem Cell Technologies), glutamine, penicillin-streptomycin, heparin $2 \mu \mathrm{g} / \mathrm{mL}$, recombinant mouse epidermal growth factor $20 \mathrm{ng} / \mathrm{mL}$, and recombinant mouse fibroblast growth factor $10 \mathrm{ng} / \mathrm{mL}$.

\section{Molecular MRI-based detection of PBT cells}

BSG D10 cells were grown in T75 flasks until $~ 80 \%$ confluence. The cells were blocked with $1 \%$ bovine serum albumin before addition of the nanoparticles. After the blocking step, the cells were incubated with MnPB-A488, MnPB-A488$\mathrm{AbC}$, or MnPB-A488-ANG2 for a further hour. The cells were rinsed three times with $10 \mathrm{~mL}$ of phosphate-buffered saline to remove any nonspecifically bound nanoparticles, trypsinized, and collected in a microcentrifuge tube at 2,100 rpm for 5 minutes. Finally, the cells were fixed with $10 \%$ formaldehyde in neutral buffer. After discarding the supernatant, each cell pellet was redispersed in $200 \mu \mathrm{L}$ of Milli-Q water and pipetted into individual microcentrifuge tubes. A solution of $0.2 \mathrm{~mL}$ agarose (1\%) was added to each tube and the tube contents were mixed thoroughly. The phantom comprised of sample-containing microcentrifuge tubes was placed next to a $2 \%$ solid agar block $\left(150 \mathrm{~cm}^{3}\right)$, secured, and measured as described previously. For each of the sequences, images were acquired at the identical coronal $0.5 \mathrm{~mm}$ thick slice, positioned at mid-height of the tubes. A T1 spin echo sequence was used for T1-weighted images (repetition time $650 \mathrm{msec}$; echo time $11 \mathrm{msec}$; echo train 1; matrix size $320 \times 256$, field of view $10 \times 10 \mathrm{~cm}^{2}$, flip angle $90^{\circ}$ ). T2-weighted images were acquired with a T2 fast relaxation fast spin echo sequence (repetition time 3,000 msec; echo time $101 \mathrm{msec}$; echo train 28 ; matrix size $384 \times 288$, field of view $10 \times 10 \mathrm{~cm}^{2}$ ). For easier reading, the original gray scale images were converted into a color scale image. Normalized intensity was derived after subtracting the signal contribution from the agarose solution.

\section{Fluorescence-based detection of PBT cells}

BSGD10 cells were seeded on poly-L-lysine and laminin-coated chamber slides and grown under the culture conditions specified above. The cells were rinsed with phosphatebuffered saline, fixed in $10 \%$ formaldehyde in neutral buffer solution for 15 minutes, and permeabilized in $0.1 \%$ Triton X-100 for a further 10 minutes. The cells were then blocked in $1 \%$ bovine serum albumin for one hour, followed by incubation with MnPB-A488, MnPB-A488$\mathrm{AbC}$, or MnPB-A488-ANG2 nanoparticles for one additional hour. The cells were then rinsed three times with phosphate-buffered saline and immunostained for GFAP using anti-GFAP antibody (Abcam, Cambridge, UK) and Alexa-647-labeled secondary antibody (Life Technologies). For visualizing nuclei, the cells were briefly stained with 4',6-diamidino-2-phenylindole (Life Technologies) and imaged using a laser scanning confocal microscope (Zeiss, Oberkochen, Germany) and ZEN 2009 software. Flow cytometry analysis of MnPB-A488, MnPB-A488-AbC, and 
MnPB-A488-ANG2 nanoparticle specificity for BSG D10 was performed as described previously. ${ }^{26}$

\section{Biodistribution of nanoparticles in a mouse model of PBT}

The mouse studies were conducted in accordance with the protocols approved by the Children's National Medical Center's Institutional Animal Care and Use Committee (292-12-05, March 2013, and 01335, March 2014). The orthotopic mouse model of PBT was developed by injecting 2-day-old BALB/c mice (The Jackson Laboratory, Bar Harbor, ME, USA) with neurospheres derived from a genetically engineered mouse model of BSG. ${ }^{43}$ The injected mice developed PBTs by 21 days post-injection. To evaluate the brain and organ biodistribution of the nanoparticles, we injected MnPB-ATxRd-transferrin into the tail veins of mice with PBT. As controls, we utilized PBT mice that did not undergo nanoparticle injections. The mice were humanely euthanized at various time points (one, 3, 6, and 24 hours post-injection; controls were sacrificed at 3 hours) using carbon dioxide and cervical dislocation in accordance with the approved protocol. Organs, including the brain, liver, kidney, spleen, and heart, were extracted from the experimental and control mice, and fixed using $10 \%$ formaldehyde in neutral buffer. The fluorescence intensities of the various organs were measured using an IVIS Spectrum preclinical in vivo imaging system (PerkinElmer, Boston, MA, USA). Mouse brains that had a positive fluorescence signal were fixed in $10 \%$ formaldehyde in neutral buffer. Sagittal sections of the brains were stained with hematoxylin and eosin to detect the presence of tumor (hypercellularity).

\section{Statistical analysis}

The mean \pm standard deviation value for each group is presented in this study. Statistical significance between groups was analyzed using Student's $t$-tests and one-way analysis of variance. A $P$-value $<0.05$ was considered to be statistically significant.

\section{Results}

\section{Synthesis and characterization of MnPB nanoparticles}

The MnPB nanoparticles were prepared using a single-pot, aqueous phase synthesis by addition of iron (II) chloride to a mixture containing manganese chloride and potassium hexacyanoferrate (III). The resulting MnPB nanoparticles had a mean size of $33 \pm 7 \mathrm{~nm}$, measured using high-resolution TEM (Figures 2A and S1), and the nanoparticle lattice parameters corresponded to Prussian blue measured by selected area electron diffraction patterns (Figure S2). FTIR spectral measurements of the MnPB nanoparticles yielded a broad band at $2,070 \mathrm{~cm}^{-1}$, corresponding to the $\mathrm{Fe}^{\mathrm{II}}-\mathrm{CN}-\mathrm{Fe}^{\mathrm{III}}$ cyanide stretch energy that was also obtained in the spectrum of Prussian blue (without manganese; Figure 2B). The FTIR spectrum for MnPB did not display a band at $2,149 \mathrm{~cm}^{-1}$ characteristic of $\mathrm{Mn}^{\mathrm{II}}-\mathrm{NC}-\mathrm{Fe}^{\mathrm{III}}{ }^{44}$

We determined the chemical composition of the MnPB nanoparticles using energy-dispersive $\mathrm{X}$-ray spectroscopy and elemental analysis (Figure $\mathrm{S} 3$ and Table S2). These analyses derived the formula $\mathrm{K}_{0.6} \mathrm{Mn}_{0.7} \mathrm{Fe}_{4}^{\mathrm{III}}\left[\mathrm{Fe}^{\mathrm{II}}(\mathrm{CN})_{6}\right]_{3.5} \cdot 3 \mathrm{H}_{2} \mathrm{O}$ for $\mathrm{MnPB}$ with an average of 0.7 atoms of manganese per unit cell. X-ray diffraction studies of MnPB and Prussian blue nanoparticle powders yielded several groups of peaks that corresponded to Prussian blue lattices (space group Fm-3m; No. 225), confirming the presence of a single phase in both types of nanoparticle constituted by Prussian blue. No mixed phase peaks were detected, suggesting that both $\mathrm{MnPB}$ and Prussian blue were made up of the Prussian blue lattice (Figure S4).

We serially diluted Prussian blue and MnPB nanoparticles to determine their longitudinal and transverse
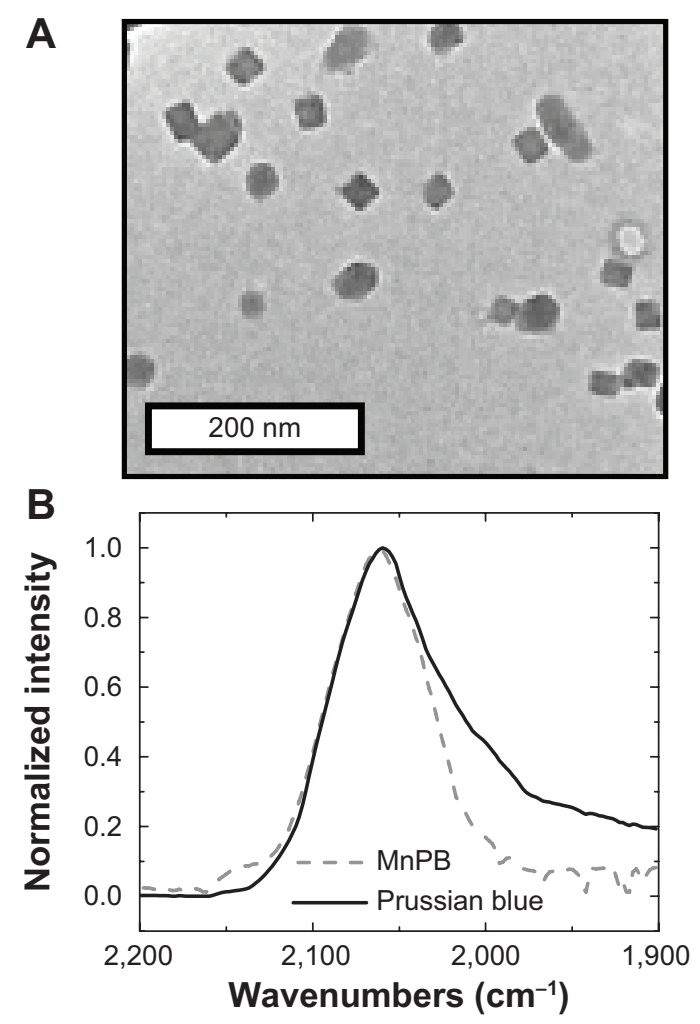

Figure 2 Properties of the MnPB nanoparticles.

Notes: (A) Representative transmission electron microscopy image of MnPB nanoparticles. (B) Fourier transform infrared spectra of Prussian blue without interstitial manganese (solid line) and MnPB (dashed line) in the cyanide stretching region $\left(1,900 \mathrm{~cm}^{-1}\right.$ to $\left.2,200 \mathrm{~cm}^{-1}\right)$.

Abbreviation: MnPB, manganese-containing Prussian blue. 


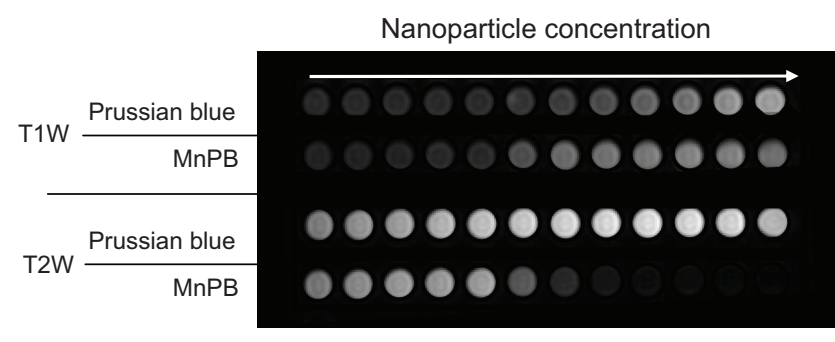

Figure 3 TIW and T2W magnetic resonance images of Prussian blue and MnPB at $3 \mathrm{~T}$.

Abbreviations: $\mathrm{TIW}, \mathrm{TI}$-weighted; $\mathrm{T} 2 \mathrm{~W}, \mathrm{~T} 2$-weighted;MnPB, manganese-containing Prussian blue.

relaxivities ( $r_{1}$ and $r_{2}$ values, respectively) at a magnetic field strength of $3 \mathrm{~T}$ (Figures 3 and S5). Relaxivity measurements with MnPB yielded an $\mathrm{r}_{1}$ value of $15.8 \mathrm{mM}^{-1} \mathrm{~s}^{-1}$ and an $\mathrm{r}_{2}$ value of $143 \mathrm{mM}^{-1} \mathrm{~s}^{-1}$. Similar measurements carried out with Prussian blue (without manganese) yielded an $r_{1}$ value of $7.9 \mathrm{mM}^{-1} \mathrm{~s}^{-1}$ and an $\mathrm{r}_{2}$ value of $14.4 \mathrm{mM}^{-1} \mathrm{~s}^{-1}$. The $r_{1}$ and $r_{2}$ values for MnPB and Prussian blue are shown in Table 1.

\section{Sizing, stability, and reproducibility of biofunctionalized MnPB nanoparticles}

Our syntheses consistently yielded nanoparticles with a $\sim 5 \%-10 \%$ variation in size between batches. Using light scattering techniques, we observed a change in the zeta potential (from $-38 \mathrm{mV}$ to $-15 \mathrm{mV}$ to $-24 \mathrm{mV}$ ) and an increase in the hydrodynamic diameter of the MnPB nanoparticles (from $126 \pm 25 \mathrm{~nm}$ to $159 \pm 30 \mathrm{~nm}$ to $240 \pm 48 \mathrm{~nm}$; Figure 4A) with the addition of the individual components of the biofunctional shell, ie, from MnPB (without modifications) to MnPB-A488 (MnPB coated with A488) and MnPB-A488-ANG2 (MnPB-A488 coated with biotinylated antibody ANG2). The resulting biofunctionalized nanoparticles were stable in both water and Dulbecco's Modified Eagle's Medium (Figure 4B) for $>4$ days (110 hours) after synthesis. The error bars in Figure 4B represent the instrument error in measuring the hydrodynamic diameters of the nanoparticles. We evaluated the safety of the

Table I Magnetic resonance measurements of relaxivity of MnPB nanoparticles

\begin{tabular}{llll}
\hline Contrast & Chemical composition & \multicolumn{2}{c}{ Relaxivity $\left(\mathbf{m M}^{-1} \mathbf{s}^{-1}\right)$} \\
\cline { 3 - 4 } agent & & $\mathbf{r}_{1}$ & $\mathbf{r}_{2}$ \\
\hline MnPB & $\mathrm{K}_{0.6} \mathrm{Mn}_{0.7} \mathrm{Fe}^{\prime \prime \prime}{ }_{4}\left[\mathrm{Fe}{ }^{\prime \prime}(\mathrm{CN})_{6}\right]_{3.5} \cdot 3 \mathrm{H}_{2} \mathrm{O}$ & 15.8 & 143.0 \\
Prussian blue & $\mathrm{K}_{2.8} \mathrm{Fe}^{\mathrm{II \prime}}{ }_{4}\left[\mathrm{Fe}^{\prime \prime}(\mathrm{CN})_{6}\right]_{3.7} \cdot 2 \mathrm{H}_{2} \mathrm{O}$ & 7.9 & 14.4 \\
\hline
\end{tabular}

Note: Magnetic resonance measurements of relaxivity were performed at $127 \mathrm{mHz}(3 \mathrm{~T})$.

Abbreviation: MnPB, manganese-containing Prussian blue.
A

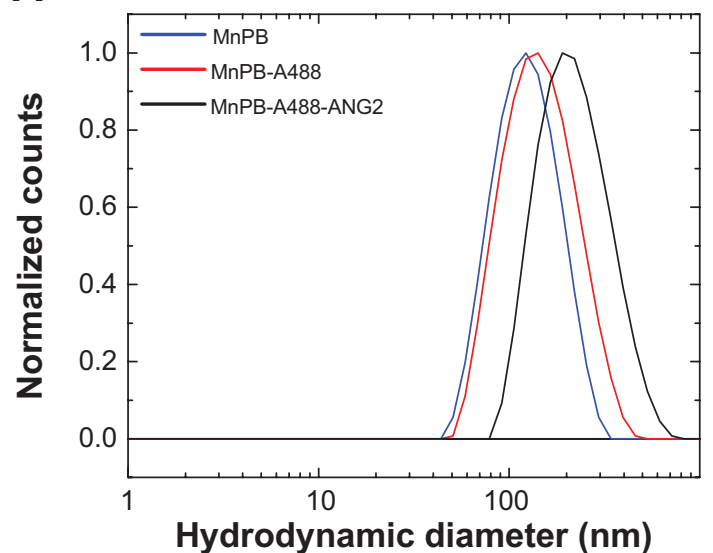

B

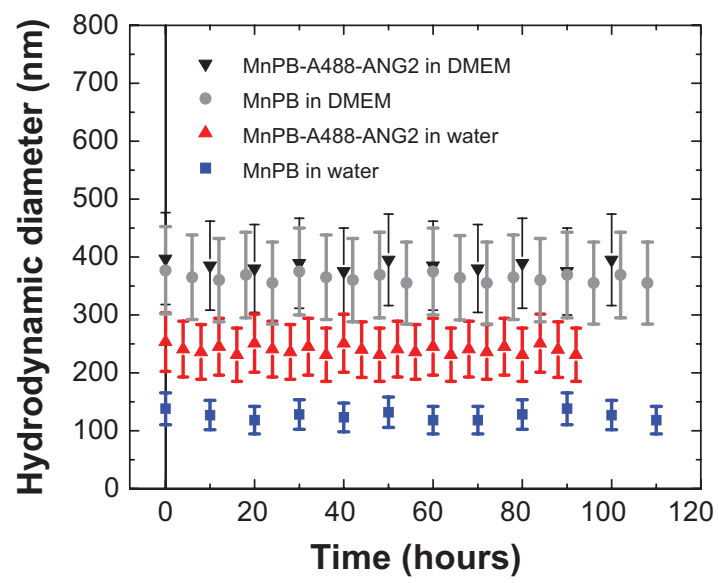

Figure 4 Size and stability of biofunctionalized MnPB nanoparticles.

Notes: (A) Size distribution of MnPB-A488-ANG2 after each functionalization step. Hydrodynamic size distributions of the MnPB nanoparticles, MnPB nanoparticles coated with avidin-Alexa Fluor 488 (MnPB-A488), and MnPB-A488 modified with biotinylated anti-neuron-glial antigen 2 (MnPB-A488-ANG2), respectively. (B) Temporal stability of MnPB nanoparticles and MnPB-A488-ANG2 in Milli-Q water and Dulbecco's Modified Eagle's Medium for up to 4 days post synthesis.

Abbreviations: MnPB, manganese-containing Prussian blue; A488, avidin-Alexa Fluor 488; ANG2, anti-neuron-glial antigen 2 antibody; DMEM, Dulbecco's Modified Eagle's Medium.

MnPB-A488-ANG2 nanoparticles as a molecular imaging agent by conducting cytotoxicity studies. These studies indicated negligible cytotoxicity at nanoparticle concentrations of $<1.25 \times 10^{-7} \mathrm{mg} /$ cell at 24 and 48 hours (Figure S6).

\section{In vitro molecular MRI-based detection of PBT cells}

To evaluate MnPB-A488-ANG2 nanoparticles as molecularly-targeted MRI agents for detecting PBT cells, we contacted BSG D10 cells with MnPB-A488-ANG2 (n=6), with MnPB-A488-AbC (n=3), and with MnPB-A488 (n=3); we then scanned the sample-containing phantoms, and obtained T1-weighted and T2-weighted images (Figure 5). The MnPBA488-ANG2 nanoparticles bound to the BSG D10 cells and generated hyperintensity (augmentation of the signal) in 


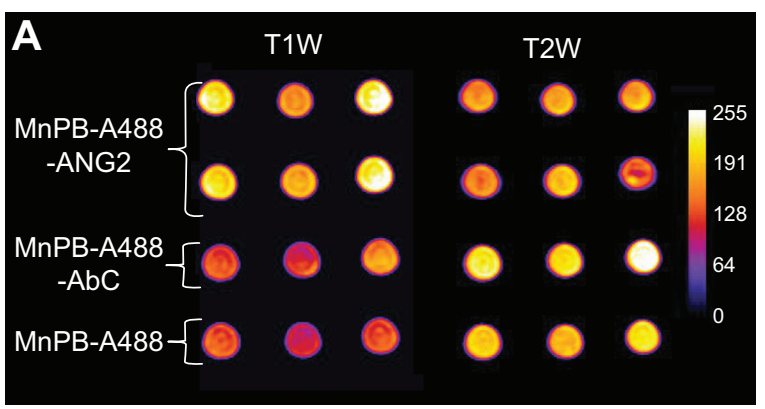

B

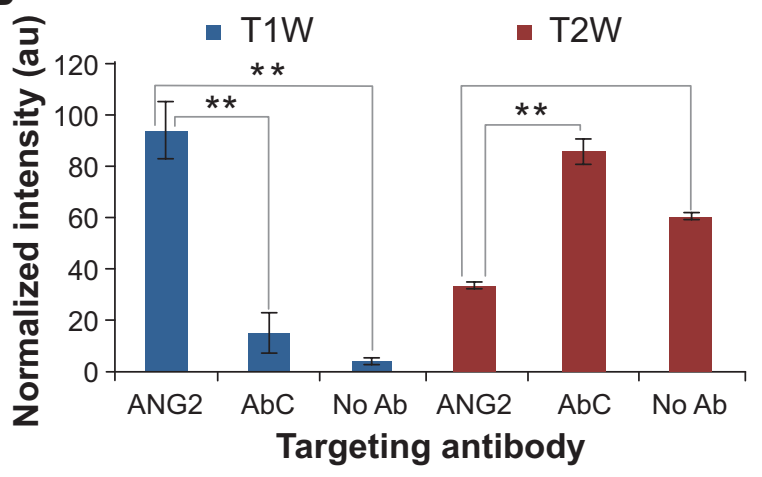

Figure 5 Molecular MRI-based detection of PBT cells using biofunctionalized Prussian blue nanoparticles. (A) TI-weighted and T2-weighted contrast enhancement in phantoms comprised of a fixed number of BSG DI0 treated with MnPB-A488ANG2 $(n=6)$, MnPB-A488-AbC $(n=3)$, or MnPB-A488 (no antibody, triplicate). (B) Normalized fluorescence signal intensity (au) for BSG DI0 treated with ANG2, $\mathrm{AbC}$, and no antibody-modified MnPB-A488. $* * P<0.05$.

Abbreviations: MnPB, manganese-containing Prussian blue; A488, avidin-Alexa Fluor 488; ANG2, anti-neuron-glial antigen 2; AbC, eotaxin antibody; BSG, brainstem glioma; TIW, TI-weighted; T2W, T2-weighted; PBT, pediatric brain tumor; au, arbitrary units; $\mathrm{Ab}$, antibody; MRI, magnetic resonance imaging.

the T1-weighted images and hypointensity (attenuation of the signal) in the T2-weighted images (Figure 5A). Control (MnPB-A488-AbC [control antibody] and MnPB-A488 [no antibody]) nanoparticles exhibited negligible binding to BSG D10 and the observed changes in magnetic resonance signal intensity were modest (Figure 5A). Quantitative analysis of the T1-weighted images showed that the MnPB-A488ANG2 nanoparticles bound to BSG D10 and were distinctly detected (signal intensity of $94 \mathrm{au}$ ). The controls (MnPBA488-AbC and MnPB-A488) showed background contrast levels, ie, a signal intensity of $\sim 10$ au (Figure 5B). Signal intensity quantification of the T2-weighted images confirmed that MnPB-A488-ANG2 nanoparticles specifically bound to BSG D10 as it generated hypointensity (signal intensity of $41 \mathrm{au}$ ). The controls (MnPB-A488-AbC and MnPB-A488) did not display significant attenuation of the signal (107 and 75, respectively; Figure 5B). These results demonstrated the feasibility of detecting PBT cells using our biofunctionalized MnPB nanoparticles in both T1-weighted and T2-weighted images.

\section{In vitro fluorescence-based detection of PBT cells}

To investigate our nanoparticles as fluorescence imaging agents, we added fixed amounts of MnPB-A488-ANG2 (containing ANG2 antibody), MnPB-A488-AbC (with control antibody $\mathrm{AbC}$ ), and $\mathrm{MnPB}-\mathrm{A} 488$ (no antibody) to BSG D10 cells. Confocal fluorescence microscopy demonstrated that the MnPB-A488-ANG2 specifically targeted BSG D10 cells (Figure 6). Control (MnPB-A488-AbC and MnPBA488) nanoparticles showed negligible binding to $\mathrm{BSG}$ D10 (negligible green fluorescence; Figure 6A and 6B).

We quantitatively measured the binding of MnPB-A488ANG2 to BSG D10 using flow cytometry. We separately added MnPB-A488-ANG2 and controls (MnPB-A488-AbC and MnPB-A488) to cultures of BSG D10 and measured the distribution of the cell populations that were fluorescently labeled (denoted as Alexa Fluor 488-positive; Figures 7A and S7). The MnPB-A488-ANG2 nanoparticles specifically bound to the BSG D10 cells ( $81 \% \pm 4 \%$ Alexa Fluor 488-positive;
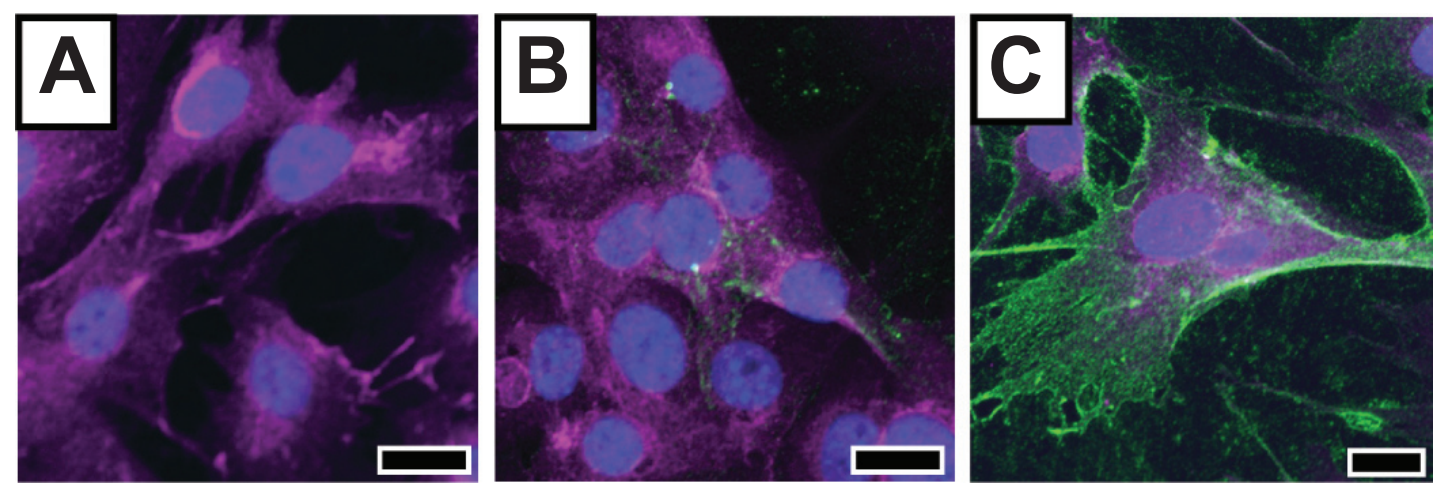

Figure 6 Fluorescence-based detection of PBT cells using biofunctionalized Prussian blue nanoparticles.

Notes: Fluorescent image of BSG DI0 treated with control nanoparticles, ie, (A) MnPB-A488 and (B) MnPB-A488-AbC, and experimental nanoparticles, ie, (C) MnPB-A488ANG2. The green fluorescence comes from A488 on the constructs. Scale bars represent $20 \mu \mathrm{m}$.

Abbreviations: BSG, brainstem glioma; MnPB, manganese-containing Prussian blue; A488, avidin-Alexa Fluor 488; ANG2, anti-neuron-glial antigen 2; AbC, eotaxin antibody; PBT, pediatric brain tumor. 


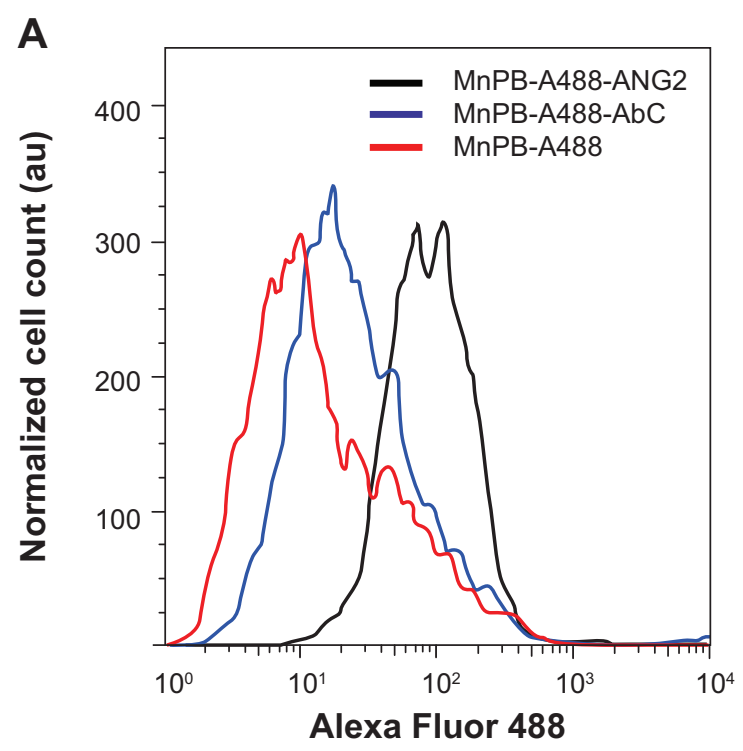

B

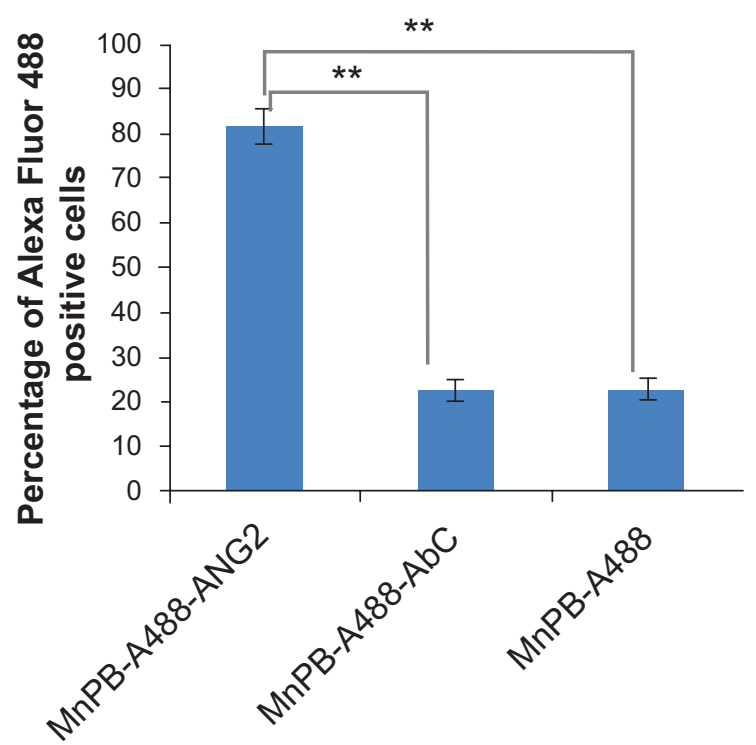

Figure 7 Flow cytometric analysis of the specificity of biofunctionalized MnPB nanoparticles for PBT cells.

Notes: (A) Representative histograms of cell count plotted against Alexa Fluor 488 detection levels for BSG DI0 cells treated with MnPB-A488 (no antibody, red line), MnPB-A488-AbC (blue line), and MnPB-A488-ANG2 (black line), and stained with 7-AAD. (B) Percentage Alexa Fluor 488-positive cells (fluorescence intensity cutoff 50) cells for BSG DI0 treated with MnPB-A488-ANG2, MnPB-A488-AbC, or MnPB-A488. $* * P<0.05$.

Abbreviations: BSG, brainstem glioma; 7-AAD, 7-aminoactinomycin D; MnPB, manganese-containing Prussian blue; A488, avidin-Alexa Fluor 488; ANG2, antineuron-glial antigen 2; PBT, pediatric brain tumor; au, arbitrary units.

Figure 7B) compared with MnPB-A488-AbC $(23 \% \pm 2 \%$ Alexa Fluor 488-positive; Figure 7B) and MnPB-A488 $(22 \% \pm 3 \%$ Alexa Fluor 488-positive; Figure 7B) where the nanoparticles showed background levels of binding to BSG D10 cells. Taken together, these results demonstrated the ability of our nanoparticles (with antibody ANG2) to molecularly target BSG D10 cells effectively using fluorescence imaging.

\section{Ex vivo nanoparticle biodistribution studies}

In order to investigate the feasibility of our nanoparticles as imaging agents in vivo, we conducted preliminary fluorescence biodistribution studies. Mice with PBTs were intravenously administered MnPB-ATxRd-transferrin nanoparticles. We made two changes to the nanoparticle design: we chose transferrin as the targeting ligand on the nanoparticles since our mice with PBTs were observed to overexpress the transferrin receptor compared with neuronglial antigen 2 (from our unpublished proteomic analysis of mice PBTs); and we selected Texas Red as the imaging fluorophore because tissue penetration depths are increased and tissue autofluorescence is decreased as the selected imaging fluorophore wavelengths are red-shifted toward near infrared wavelengths. Following intravenous injection, the mice organs, ie, brain, liver, kidney, spleen and heart, were harvested at different time points (between one and 24 hours) and their fluorescence measured.

Fluorescence measurements of the brains of the mice detected the nanoparticles in the brain between one hour and 3 hours, with the fluorescent signal reaching a maximum at one hour (Figure 8A). Little to no fluorescence was detected after 3 hours and in the control mouse brain (without nanoparticle injection). Fluorescence organ biodistribution measurements showed that the nanoparticles were cleared via the liver, kidney, and spleen (Figure 8B and 8C). Mouse brains that were positive for fluorescent signal were analyzed by histology. Sagittal sections of the brain stained by hematoxylin and eosin revealed a hypercellular region located within the fluorescently active brain segment localized in the ventricular and periventricular regions of the mouse brain (Figure 9). These results indicated the feasibility of utilizing our nanoparticles for fluorescence imaging of PBTs in vivo.

\section{Discussion}

Here we have described the synthesis of novel MnPB nanoparticles by utilizing the cation-incorporating property (for charge neutrality) of Prussian blue nanoparticles. Comprehensive physical and chemical characterization (Figures 2 and S1-S4) of these nanoparticles using TEM, energy-dispersive X-ray spectroscopy, powder X-ray diffraction, and FTIR confirmed that they have a structure virtually identical to that of Prussian blue nanoparticles, indicating that the $\mathrm{Mn}^{2+}$ ions were not modifying the Prussian blue lattice structure and likely occupying the lattice tetragonal sites (interstitial vacancies). MnPB nanoparticles possess MRI 

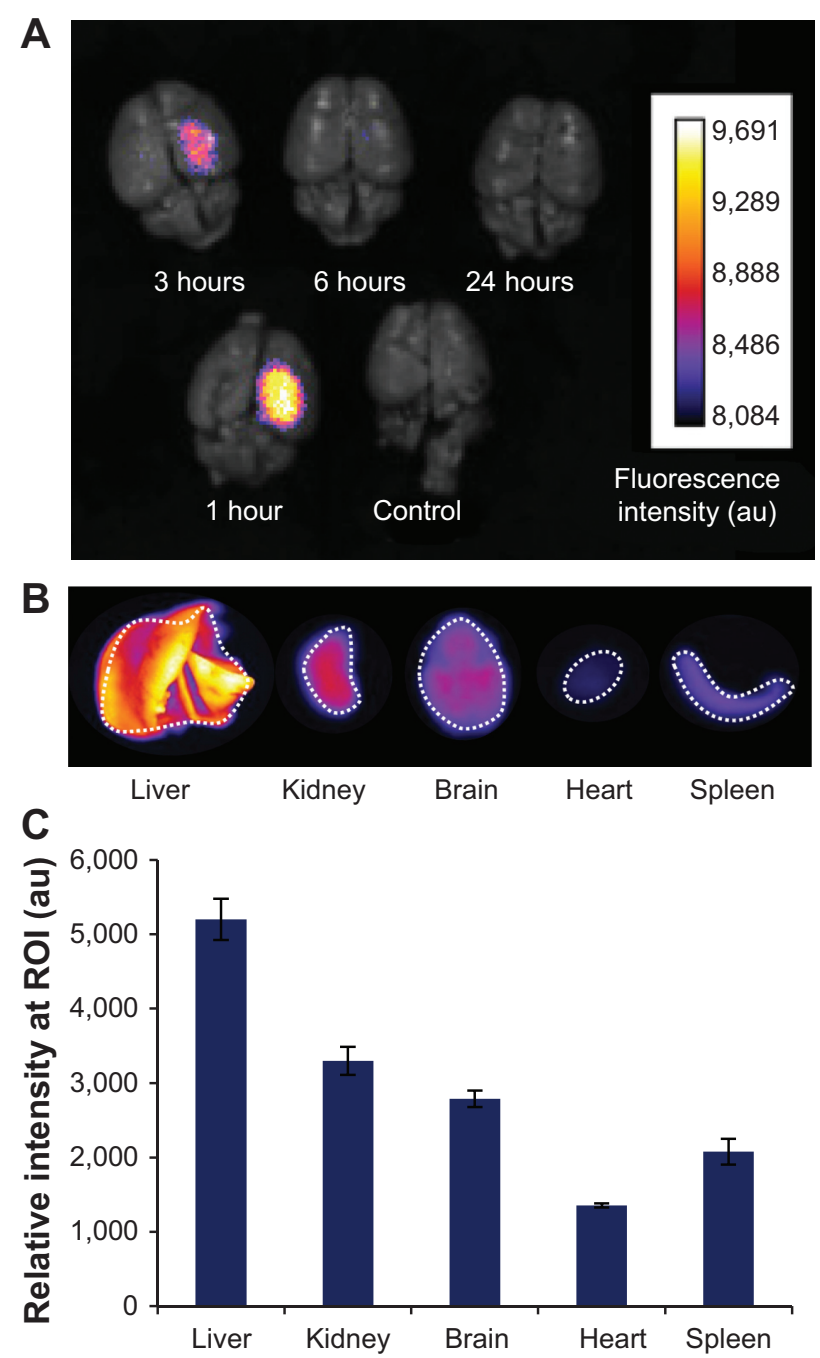

Figure 8 Ex vivo fluorescence imaging of biodistribution of the nanoparticles in an orthotopic mouse model of PBT.

Notes: (A) Fluorescent signal observed in the brains of four separate mice with PBT ( $I-24$ hours) injected intravenously (via tail vein) with transferrin-coated Prussian blue nanoparticles (MnPB-ATxRd-Tf). A control mouse with PBT was not injected with nanoparticles. (B) Representative ex vivo fluorescence imaging of organ biodistribution of the nanoparticles at 3 hours post-injection. (C) Histograms quantifying the observed fluorescence biodistribution of the nanoparticles 3 hours post-injection. ROls for intensity measurements are indicated by white dashed lines in (B).

Abbreviations: PBT, pediatric brain tumor; MnPB, manganese-containing Prussian blue; ATxRd, Texas Red-labeled avidin; Tf, transferrin; ROI, region of interest; au, arbitrary units.

contrast-enhancing capabilities (both negative and positive), which was confirmed by generation of positive contrast on a T1-weighted image sequence and negative contrast on a T2-weighted image sequence at $3 \mathrm{~T}$ (Figures 3 and S4, Table 1). Compared with $\mathrm{Gd}^{3+}$-containing commercial contrast agents, which are typically used solely as positive contrast agents, the magnetic measurement data established the efficiency of the MnPB nanoparticles as MRI contrast agents useful in both T1-weighted and T2-weighted sequences, thus confirming MnPB nanoparticles as versatile contrast agents in new imaging applications involving combinations

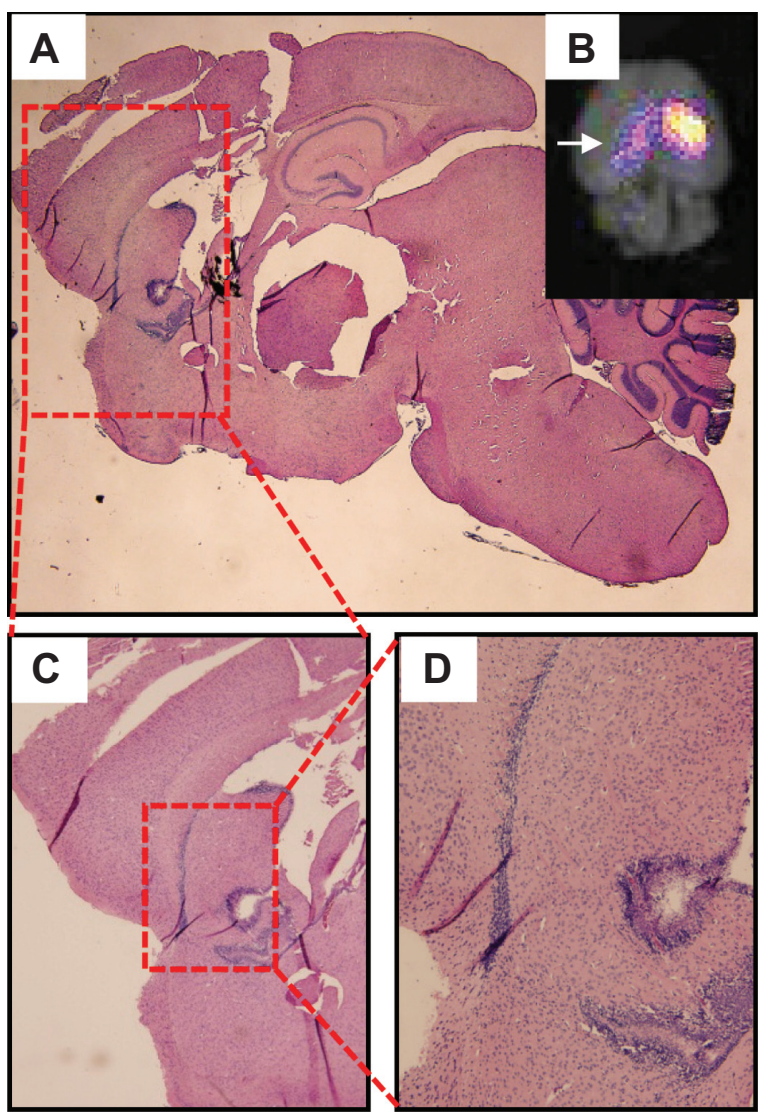

Figure 9 Histological analysis of fluorescence-positive regions of mice brains with PBTs.

Notes: (A) Sagittal slice of mouse brain stained with hematoxylin and eosin. (B) Inset showing a fluorescence image of the brain, with arrow indicating the viewing direction of the sagittal section in (A). (C, D) Progressive zooms of the hematoxylin and eosin-stained sagittal brain sections containing a hypercellular ventricular and periventricular region within the fluorescence-positive region.

Abbreviation: PBT, pediatric brain tumor.

of both T1-weighted and T2-weighted sequences. Preliminary studies confirm the feasibility of utilizing MnPB as MRI contrast agents in both T1-weighted and T2-weighted sequences (Figure $\mathrm{S} 8$ ).

The surface of the MnPB nanoparticles was negatively charged (zeta potential $-38 \mathrm{mV}$ ), which electrostatically stabilized the nanoparticle suspension, thereby eliminating the need for stabilizing surfactants. ${ }^{45,46}$ To biofunctionalize the nanoparticles for molecular targeting of PBTs, the MnPB nanoparticles were first coated with a layer of fluorescent avidin (A488 or ATxRd). Avidin, which is a highly positively charged glycoprotein at physiological $\mathrm{pH}$ (isoelectric point $\sim 10.5$ ), was coated on the surface of the negatively charged MnPB nanoparticles using electrostatic selfassembly. ${ }^{15,17}$ The surfaces of the fluorescent avidin-coated MnPB nanoparticles were modified with the PBT-targeting or control ligands (ANG2, transferrin, or $\mathrm{AbC}$ ) via an avidin-biotin interaction, which is one of the most robust, 
noncovalent interactions identified $\left(\mathrm{K}_{\mathrm{d}}=10^{-15} \mathrm{M}\right)$, and is capable of sustaining extremes of temperature, $\mathrm{pH}$, and harsh chemical conditions. ${ }^{15,33,47,48}$ The biofunctionalized nanoparticles demonstrated temporal stability (over 4 days), both in water and in Dulbecco's Modified Eagle's Medium (Figure 4), and negligible cytotoxicity (at concentrations $<1.25 \times 10^{-7} \mathrm{mg} /$ cell; Figure S6) to PBT cells (BSG D10), indicating their safety for use as molecular imaging agents. The nanoparticles were able to specifically detect PBT cells (BSG D10) using MRI in both T1-weighted and T2-weighted sequences by generating specific positive and negative contrast, respectively, relative to the controls that exhibited background contrast (Figure 5). The nanoparticles also demonstrated specific fluorescence-based detection of PBT cells (Figures 6 and 7, and S7). These results validated the utility of our nanoparticles as multimodal imaging agents for PBTs.

Preliminary biodistribution studies using ex vivo fluorescence imaging in a mouse model of PBT indicated that the nanoparticles were cleared via the liver, kidney, and spleen (Figure 8). Longer circulation times and lower immunogenicity of our nanoparticles for future in vivo imaging studies will require modifications of the current design, including coating of the nanoparticles with the "stealth" polymer, polyethylene glycol (PEGylation), ${ }^{18,49,50}$ which is currently being investigated. Ex vivo brain fluorescence biodistribution studies also demonstrated that the fluorescent signal of our nanoparticles was detected in the brain between one hour and 3 hours. Histological analysis of the fluorescent region within the mouse brain confirmed the presence of a hypercellular region in the ventricular and periventricular regions overlapping the region of the brain exhibiting a fluorescent signal (Figure 9). The increased fluorescence signal in the brain was possibly due to an increased transient presence of our nanoparticles in the hypercellular region within the brain (Figure 9). For future in vivo imaging of PBTs, the design of our nanoparticles will be modified to incorporate ligands (by varying type of ligand, ligand surface density) that cross the blood-brain barrier, penetrate the brain parenchyma, and assist uptake within PBTs. ${ }^{51,52}$ These studies are currently in progress.

\section{Conclusion}

In this paper, we describe the synthesis and characterization of novel, biofunctionalized MnPB nanoparticles for imaging PBTs. These nanoparticles exhibited fluorescence as well as superior and versatile MRI contrast enhancement capabilities. We demonstrated these nanoparticles to be multimodal imaging agents using MRI and fluorescence-based detection of PBT cells in vitro. Preliminary ex vivo fluorescence imaging provided a proof-of-concept demonstration of our nanoparticles as imaging agents and suggests the potential of these nanoparticles as agents for multimodal and molecular imaging of PBTs in vivo.

\section{Acknowledgments}

This work was supported by the Sheikh Zayed Institute for Pediatric Surgical Innovation (Research Advisory Committee Award 30000174). The authors acknowledge the support of The Maryland NanoCenter at University of Maryland and its NispLab. The NispLab is supported in part by the National Science Foundation as a Materials Research Science and Engineering Center Shared Experimental Facility. The authors gratefully acknowledge the following personnel: S Taylor from the Department of Chemistry at University of Maryland Optical Instrumentation Facility for the FTIR spectroscopy; D Taylor and PY Zavalij at the X-ray Crystallographic Center, Department of Chemistry and Biochemistry, University of Maryland, for experimental data and analysis; L Chakrabarti from the Children's National Medical Center for assistance with flow cytometry studies; and Paul C Wang and Stephen Lin from the Molecular Imaging Laboratory at Howard University College of Medicine for their assistance with the ex vivo fluorescence imaging studies.

\section{Disclosure}

The authors report no conflicts of interest in this work.

\section{References}

1. Mostoufi-Moab S, Grimberg A. Pediatric brain tumor treatment: growth consequences and their management. Pediatr Endocrinol Rev. 2010;8(1):6-17.

2. Pollack I. Multidisciplinary management of childhood brain tumors: a review of outcomes, recent advances, and challenges. $J$ Neurosurg Pediatr. 2011;8(2):135-148.

3. Jansen MH, Kaspers GJ. A new era for children with diffuse intrinsic pontine glioma: hope for cure? Expert Rev Anticancer Ther. 2012;12(9):1109-1112.

4. Jansen MH, van Vuurden DG, Vandertop WP, Kaspers GJ. Diffuse intrinsic pontine gliomas: a systematic update on clinical trials and biology. Cancer Treat Rev. 2012;38(1):27-35.

5. Schwartzentruber J, Korshunov A, Liu X-Y, et al. Driver mutations in histone $\mathrm{H} 3.3$ and chromatin remodelling genes in paediatric glioblastoma. Nature. 2012;482(7384):226-231.

6. Warren KE. Diffuse intrinsic pontine glioma: poised for progress. Front Oncol. 2012;2:205.

7. Srikanth M, Kessler JA. Nanotechnology - novel therapeutics for CNS disorders. Nat Rev Neurol. 2012;8(6):307-318.

8. Prabhakar U, Maeda H, Jain RK, et al. Challenges and key considerations of the enhanced permeability and retention effect for nanomedicine drug delivery in oncology. Cancer Res. 2013;73(8): $2412-2417$.

9. Greish K. Enhanced permeability and retention (EPR) effect for anticancer nanomedicine drug targeting. In: Grobmyer SR, Moudgil BM, editors. Cancer Nanotechnology. Totowa, NJ, USA: Humana Press; 2010. 
10. Liu X, Chen Y, Li H, et al. Enhanced retention and cellular uptake of nanoparticles in tumors by controlling their aggregation behavior. ACS Nano. 2013;7(7):6244-6257.

11. Veiseh M, Gabikian P, Bahrami S-B, et al. Tumor paint: a chlorotoxin: Cy5.5 bioconjugate for intraoperative visualization of cancer foci. Cancer Res. 2007;67(14):6882-6888.

12. Veiseh O, Sun C, Fang C, et al. Specific targeting of brain tumors with an optical/magnetic resonance imaging nanoprobe across the bloodbrain barrier. Cancer Res. 2009;69(15):6200-6207.

13. Veiseh O, Gunn JW, Zhang M. Design and fabrication of magnetic nanoparticles for targeted drug delivery and imaging. Adv Drug Deliv Rev. 2010;62(3):284-304.

14. Wu C, Hansen SJ, Hou Q, et al. Design of highly emissive polymer dot bioconjugates for in vivo tumor targeting. Angew Chem Int Ed. 2011;50(15):3430-3434.

15. Goldman ER, Balighian ED, Mattoussi H, et al. Avidin: a natural bridge for quantum dot-antibody conjugates. J Am Chem Soc. 2002;124(22): 6378-6382.

16. Kreuter J. Nanoparticulate systems for brain delivery of drugs. Adv Drug Deliv Rev. 2001;47(1):65-81.

17. Jaiswal JK, Mattoussi H, Mauro JM, Simon SM. Long-term multiple color imaging of live cells using quantum dot bioconjugates. Nat Biotechnol. 2002;21(1):47-51.

18. Jokerst JV, Lobovkina T, Zare RN, Gambhir SS. Nanoparticle PEGylation for imaging and therapy. Nanomedicine (Lond). 2011;6(4) 715-728.

19. Chekina N, Horák D, Jendelová P, et al. Fluorescent magnetic nanoparticles for biomedical applications. J Mater Chem. 2011;21(21):7630.

20. Frimpong RA, Hilt JZ. Magnetic nanoparticles in biomedicine: synthesis, functionalization and applications. Nanomedicine (Lond) 2010;5(9):1401-1414.

21. Pankhurst Q, Connolly J, Jones SK, Dobson J. Applications of magnetic nanoparticles in biomedicine. J Phys Appl Phys. 2003;36(13) R167-R181.

22. Vatta LL, Sanderson RD, Koch KR. Magnetic nanoparticles: properties and potential applications. Adv Mater. 2006;78(9):1793-1801.

23. Schütz CA, Juillerat-Jeanneret L, Mueller H, Lynch I, Riediker M. Therapeutic nanoparticles in clinics and under clinical evaluation Nanomedicine (Lond). 2013;8(3):449-467.

24. Heyltex Corporation. Radiogardase ${ }^{\mathrm{TM}}$ Prussian blue insoluble capsules Katy, TX, USA: Heyltex Corporation; 2008. Available from: http:/ www.heyltex.com/radiogardase.php. Accessed April 23, 2014.

25. Faustino PJ, Yang Y, Progar JJ, et al. Quantitative determination of cesium binding to ferric hexacyanoferrate: Prussian blue. J Pharm Biomed Anal. 2008;47(1):114-125.

26. Yang Y, Faustino P, Progar J, et al. Quantitative determination of thallium binding to ferric hexacyanoferrate: Prussian blue. Int J Pharm. 2008;353(1-2):187-194.

27. Bernstein EJ, Schmidt-Lauber C, Kay J. Nephrogenic systemic fibrosis: a systemic fibrosing disease resulting from gadolinium exposure. Best Pract Res Clin Rheumatol. 2012;26(4):489-503.

28. Haylor J, Schroeder J, Wagner B, et al. Skin gadolinium following use of MR contrast agents in a rat model of nephrogenic systemic fibrosis. Radiology. 2012;263(1):107-116.

29. Langer RD, Lorke DE, Neidl van Gorkom KF, et al. In an animal model nephrogenic systemic fibrosis cannot be induced by intraperitoneal injection of high-dose gadolinium based contrast agents. Eur J Radiol. 2012;81(10):2562-2567.

30. Yang L, Krefting I, Gorovets A, et al. Nephrogenic systemic fibrosis and class labeling of gadolinium-based contrast agents by the Food and Drug Administration. Radiology. 2012;265(1):248-253.

31. Dumont MF, Hoffman HA, Yoon PR, et al. Biofunctionalized gadolinium-containing Prussian blue nanoparticles as multimodal molecular imaging agents. Bioconjug Chem. 2014;25(1):129-137.
32. Bayer EA, Wilchek M. The use of the avidin-biotin complex as a tool in molecular biology. In: Glick D, editor. Methods of Biochemical Analysis. Hoboken, NJ, USA: John Wiley \& Sons, Inc.; 2006.

33. Sun S, Huang X, Ma M, et al. Systematic evaluation of avidin-biotin interaction by fluorescence spectrophotometry. Spectrochim Acta A Mol Biomol Spectrosc. 2012;89:99-104.

34. James ML, Gambhir SS. A molecular imaging primer: modalities, imaging agents, and applications. Physiol Rev. 2012;92(2):897-965.

35. Poussaint TY, Kocak M, Vajapeyam S, et al. MRI as a central component of clinical trials analysis in brainstem glioma: a report from the Pediatric Brain Tumor Consortium (PBTC). Neuro Oncol. 2011;13(4): $417-427$.

36. Stummer W, Novotny A, Stepp H, Goetz C, Bise K, Reulen HJ. Fluorescence-guided resection of glioblastoma multiforme utilizing 5-ALA-induced porphyrins: a prospective study in 52 consecutive patients. J Neurosurg. 2000;93(6):1003-1013.

37. Sugiarto S, Persson AI, Munoz EG, et al. Asymmetry-defective oligodendrocyte progenitors are glioma precursors. Cancer Cell. 2011;20(3): 328-340.

38. Al-Mayhani MT, Grenfell R, Narita M, et al. NG2 expression in glioblastoma identifies an actively proliferating population with an aggressive molecular signature. Neuro Oncol. 2011;13(8):830-845.

39. Wang J, Svendsen A, Kmiecik J, et al. Targeting the NG2/CSPG4 proteoglycan retards tumour growth and angiogenesis in preclinical models of GBM and melanoma. PLoS One. 2011;6(7):e23062.

40. Martell LA, Agrawal A, Ross DA, Muraszko KM. Efficacy of transferrin receptor-targeted immunotoxins in brain tumor cell lines and pediatric brain tumors. Cancer Res. 1993;53(6):1348-1353.

41. Daniels TR, Delgado T, Helguera G, Penichet ML. The transferrin receptor part II: targeted delivery of therapeutic agents into cancer cells. Clin Immunol. 2006;121(2):159-176.

42. Weaver M, Laske D. Transferrin receptor ligand-targeted toxin conjugate (Tf-CRM107) for therapy of malignant gliomas. J Neurooncol. 2003;65(1):3-14.

43. Becher OJ, Hambardzumyan D, Walker TR, et al. Preclinical evaluation of radiation and perifosine in a genetically and histologically accurate model of brainstem glioma. Cancer Res. 2010;70(6):2548-2557.

44. Ohkoshi S, Matsuda T, Tokoro H, Hashimoto K. A surprisingly large thermal hysteresis loop in a reversible phase transition of $\mathrm{RbxMn}[\mathrm{Fe}(\mathrm{CN}) 6](\mathrm{x}+2) / 3 \cdot \mathrm{zH}_{2} \mathrm{O}$. Chem Mater. 2005;17(1):81-84.

45. Dumont MF, Knowles ES, Guiet A, et al. Photoinduced magnetism in core/shell Prussian blue analogue heterostructures of $\mathrm{KjNik}[\mathrm{Cr}(\mathrm{CN}) 6] 1 \cdot \mathrm{nH} 2 \mathrm{O}$ with $\mathrm{RbaCob}[\mathrm{Fe}(\mathrm{CN}) 6] \mathrm{c} \cdot \mathrm{mH} 2 \mathrm{O}$. Inorg Chem. 2011;50(10):4295-4300.

46. Catala L, Brinzei D, Prado Y, et al. Core-multishell magnetic coordination nanoparticles: toward multifunctionality on the nanoscale. Angew Chem Int Ed. 2009;48(1):183-187.

47. Fraenkel-Conrat H, Snell NS, Ducay ED. Avidin. II. Composition and mode of action of avidin A. Arch Biochem Biophys. 1952;39(1): 97-107.

48. Green NM. Avidin. 4. Stability at extremes of $\mathrm{pH}$ and dissociation into sub-units by guanidine hydrochloride. Biochem J. 1963;89:609-620.

49. Otsuka H, Nagasaki Y, Kataoka K. PEGylated nanoparticles for biological and pharmaceutical applications. Adv Drug Deliv Rev. 2003;55(3):403-419.

50. Sun C, Sze R, Zhang M, Sun C, Sze R, Zhang M. Folic acid- PEG conjugated superparamagnetic nanoparticles for targeted cellular uptake and detection by MRI. J Biomed Mater Res Part A . 2006;78(3):550-557.

51. Wiley DT, Webster P, Gale A, Davis ME. Transcytosis and brain uptake of transferrin-containing nanoparticles by tuning avidity to transferrin receptor. Proc Natl Acad Sci U S A. 2013;110(21):8662-8667.

52. Nance EA, Woodworth GF, Sailor KA, et al. A dense poly(ethylene glycol) coating improves penetration of large polymeric nanoparticles within brain tissue. Sci Transl Med. 2012;4(149):149ra119. 


\section{Supplementary materials}

Table SI Names and components of nanoparticles described in text

\begin{tabular}{llll}
\hline Construct name & Core & Fluorophore in avidin layer & Targeting ligand \\
\hline MnPB & MnPB & None & None \\
MnPB-A488 & MnPB & Alexa Fluor 488 & None \\
MnPB-ATxRd & MnPB & Texas Red & None \\
MnPB-A488-ANG2 & MnPB & Alexa Fluor 488 & Anti-neuron-glial antigen 2 \\
MnPB-A488-AbC & MnPB & Alexa Fluor 488 & Eotaxin-3 antibody \\
MnPB-ATxRd-Tf & MnPB & Texas Red & Human transferrin \\
\hline
\end{tabular}

Abbreviation: MnPB, manganese-containing Prussian blue particles.
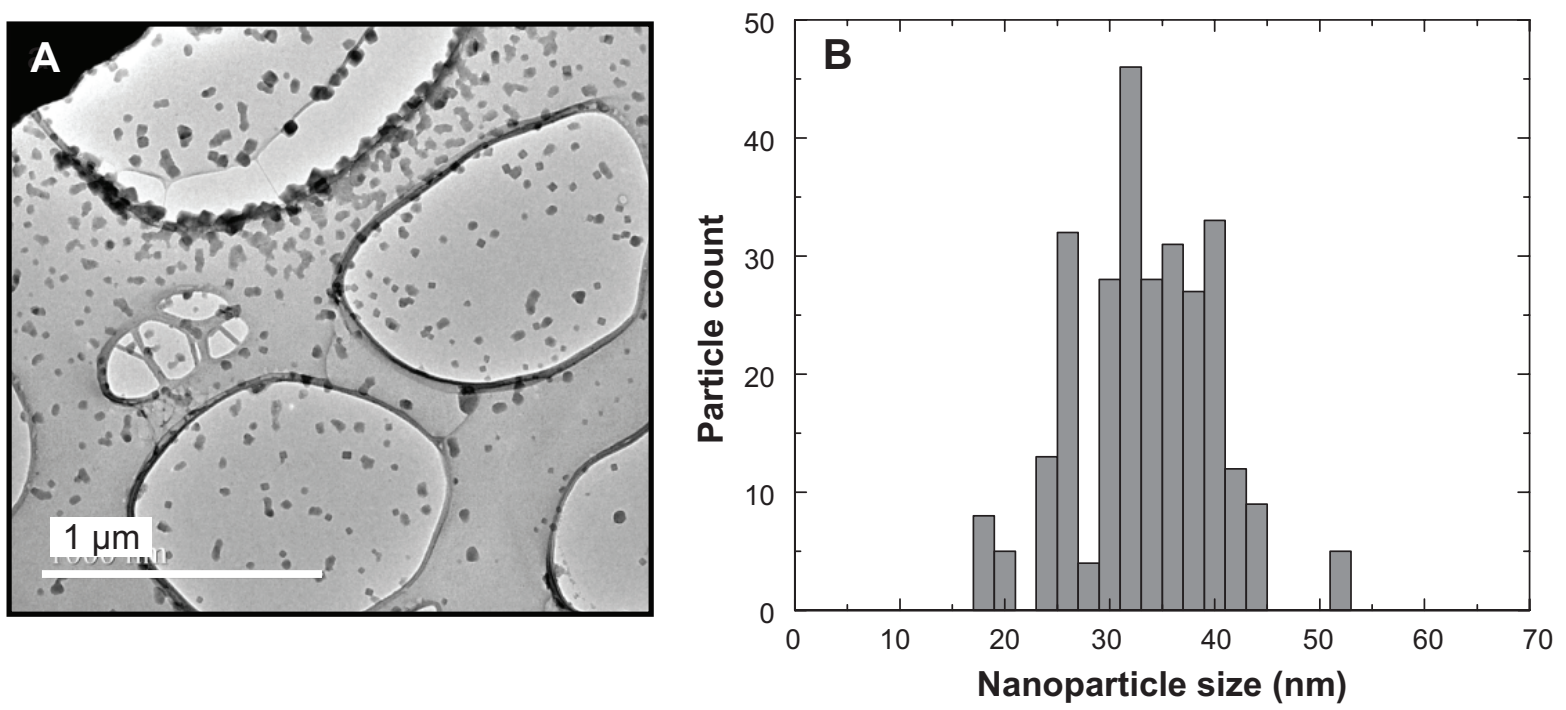

Figure SI Size of manganese-containing Prussian blue nanoparticles.

Notes: (A) Transmission electron micrograph of an oversized agglomerate consisting of over 500 nanoparticles (scale bar I $\mu \mathrm{m}$ ) used for selected area electron diffraction. (B) Histogram showing the size distribution of 198 nanoparticles with a mean nanoparticle size of $33 \pm 7 \mathrm{~nm}$. The size analysis was performed with Image software by manually measuring the size of the individual nanoparticles from the transmission electron microscopy images of well dispersed, individual nanoparticles.

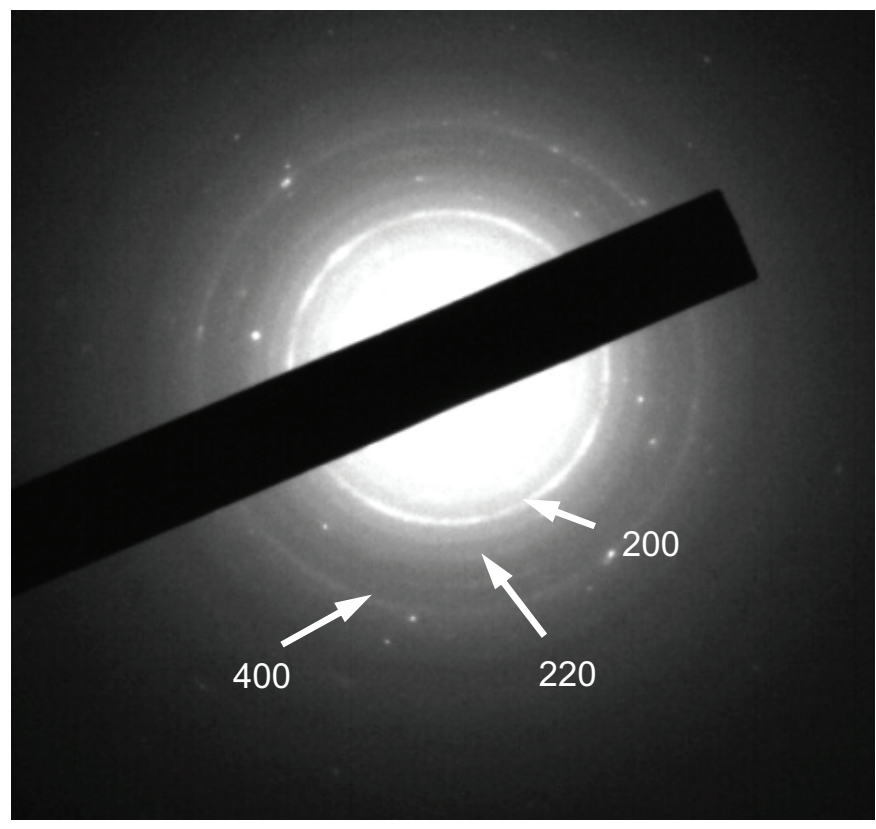

Figure S2 Selected area electron diffraction pattern of a manganese-containing Prussian blue nanoparticle with 200, 220, and 400 reflections identified as the lattice corresponding to Prussian blue. 

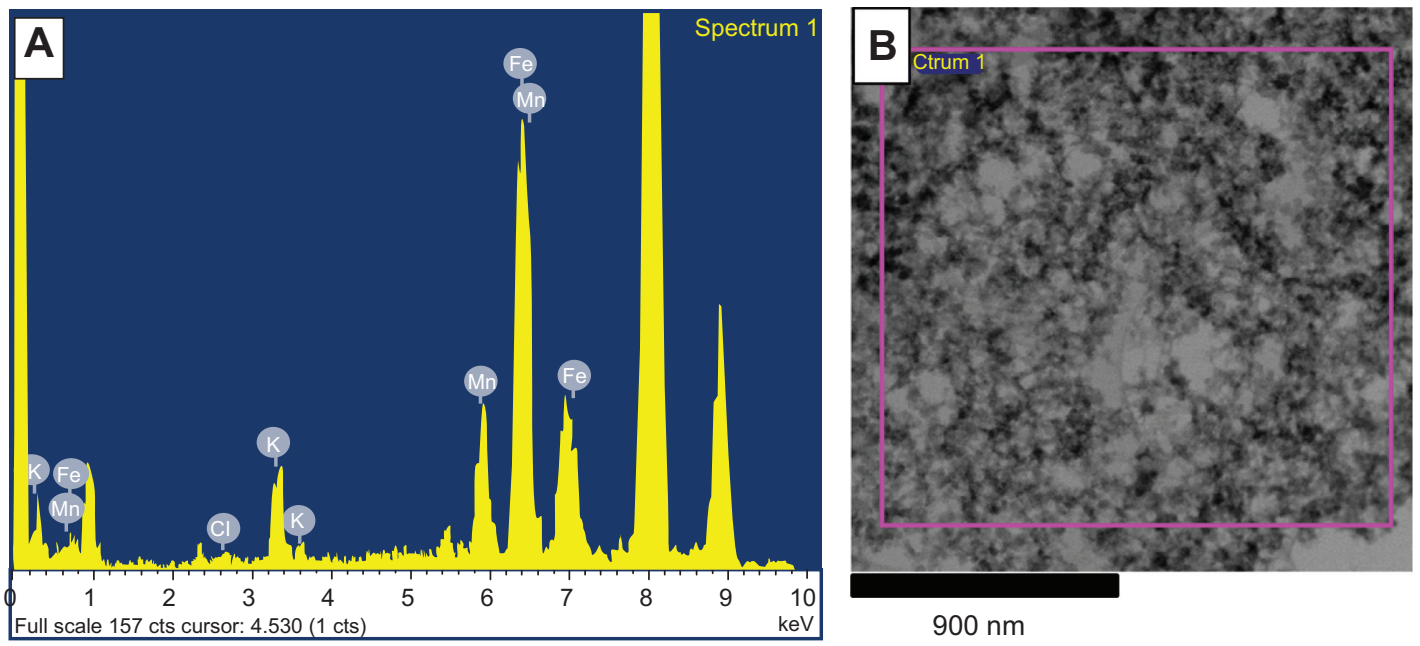

Figure S3 Composition of manganese-containing Prussian blue nanoparticles.

Notes: (A) Energy-dispersive X-ray spectrum corresponding to the region of interest shown in (B), which is a representative transmission electron micrograph of the nanoparticles. The purple line indicates the limits of a typical region of interest analyzed by energy-dispersive X-ray spectroscopy. The composition was derived by built-in software (Inca; Oxford Instruments, Abingdon, UK) from the attribution of the electronic energy profile for Fe, $\mathrm{K}$, and $\mathrm{Mn}$.

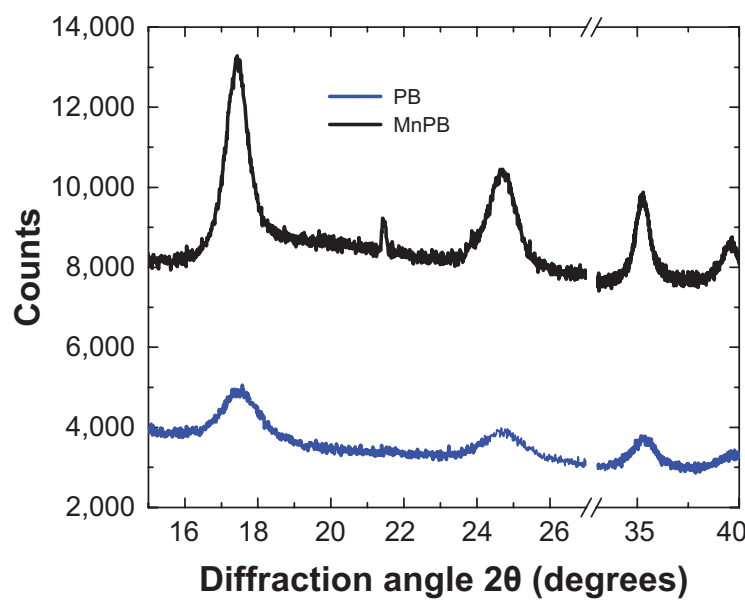

Figure S4 Room temperature X-ray diffractogram of a sample of MnPB and PB with peaks at around 17, 24, and 35 degrees, corresponding to the 200, 220, and 400 diffraction planes, respectively.

Notes: X-ray diffraction from a sample of particles showed several groups of peaks corresponding to the 200,220 , and 400 diffraction planes at 17.43, 24.67, and 35.24 degrees, respectively, for MnPB and I7.5I, 24.68, and 35.29 degrees, respectively, for PB. These diffraction peaks were indexed to Prussian blue lattices using the space group Fm-3m (No. 225) and confirmed the presence of one phase constituted by Prussian blue. Using the (400) reflections fitted to a Gaussian function, the lattice parameters for each compound were calculated. We found the following lattice constants: MnPB $(a=10.19 \AA)$ and PB $(a=10.17 \AA)$. No peaks corresponding to a mixed phase (such as manganese) with different lattice parameters were detected, suggesting that both MnPB and PB are made up of the Prussian blue lattice.

Abbreviations: PB, Prussian blue particles without interstitial gadolinium; MnPB, manganese-containing Prussian blue.

Table S2 Summary of relative percentages of potassium, iron, and manganese from three distinct energy-dispersive $X$-ray spectroscopic scans of manganese-containing Prussian blue particles

\begin{tabular}{llll}
\hline & Potassium (\%) & Iron (\%) & Manganese (\%) \\
\hline Spectrum I & 9.03 & 79.86 & 7.86 \\
Spectrum 2 & 8.53 & 85.73 & 7.32 \\
Spectrum 3 & 8.77 & 84.05 & 8.87 \\
\hline
\end{tabular}
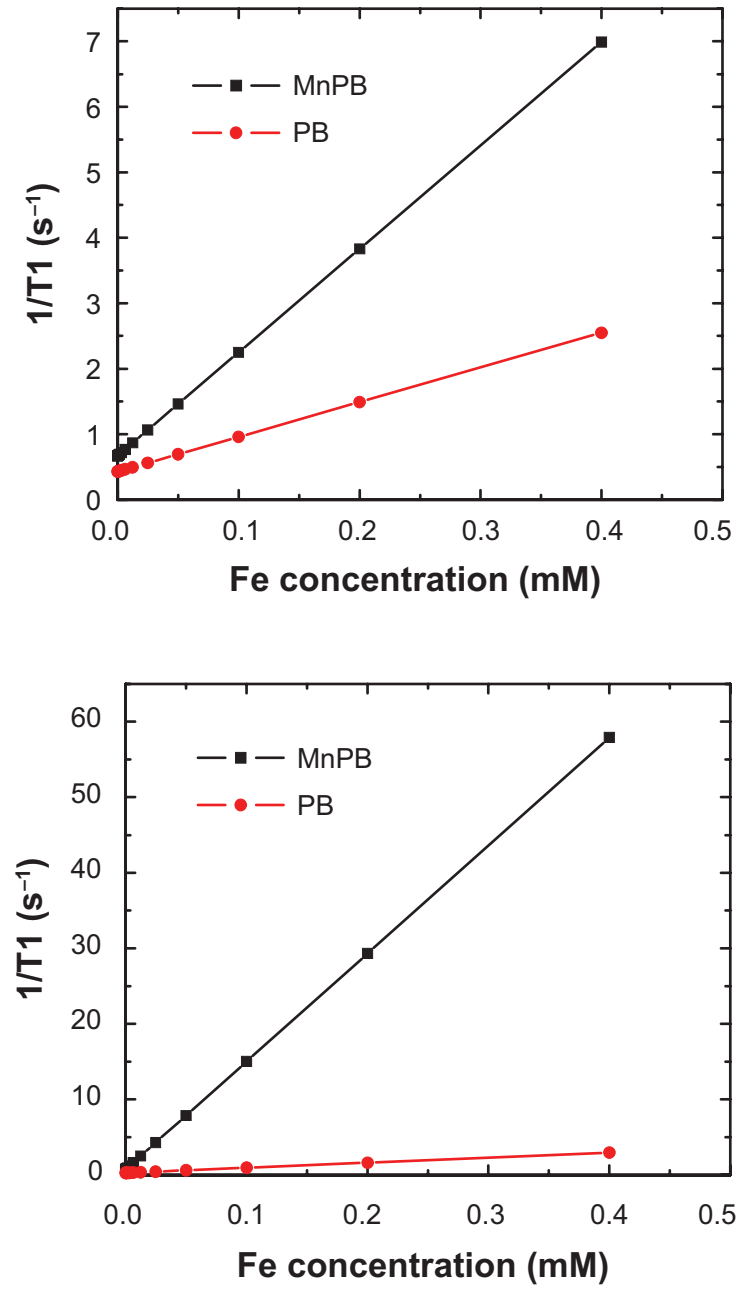

Figure S5 Inverse of relaxation times $(I / T I=R I$ and $I / T 2=R 2)$ plotted against concentrations of the main paramagnetic ion.

Note: $r_{1}$ and $r_{2}$ values are derived from the linear fitting of these plots.

Abbreviations: PB, Prussian blue particles without interstitial gadolinium; MnPB, manganese-containing Prussian blue. 


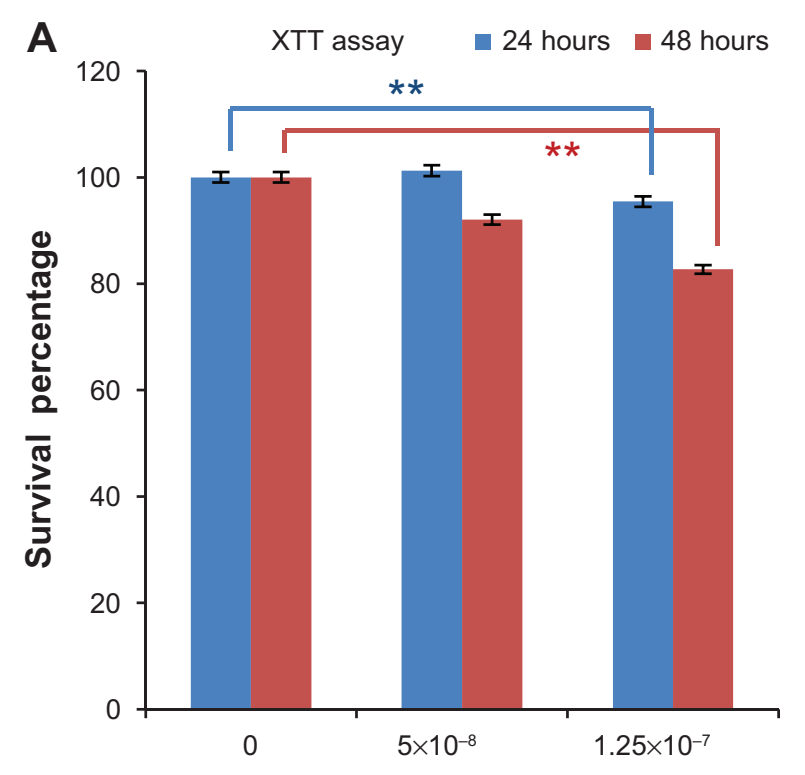

Nanoparticle concentration (mg/cell)

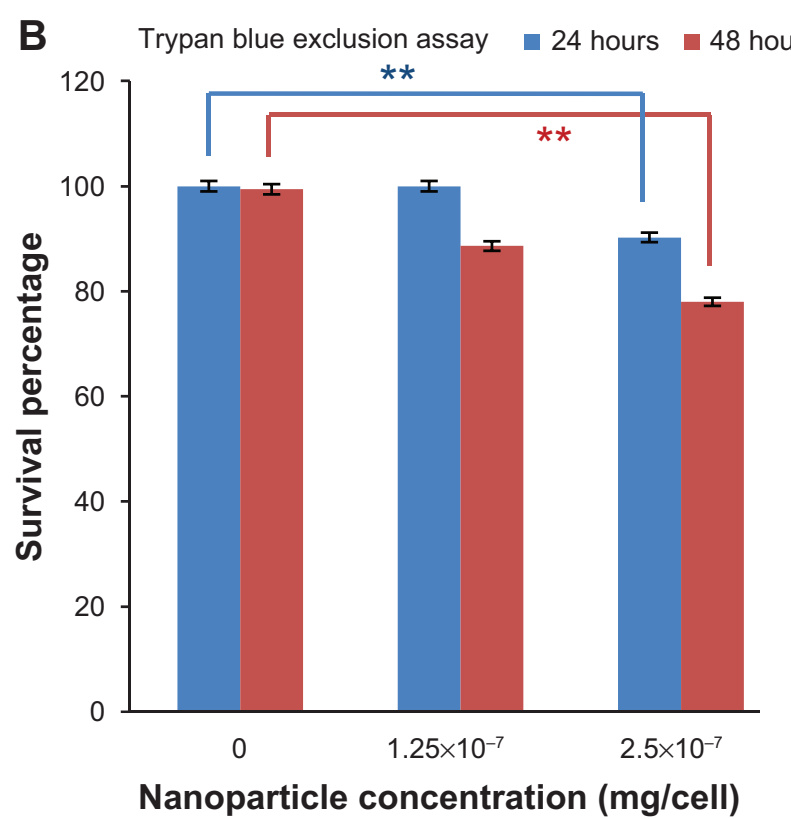

Figure S6 Cytotoxicity studies of biofunctionalized MnPB nanoparticles. Viability (survival percentage) of BSG DI0 coincubated with varying concentrations of MnPB-A488ANG2 determined by $(\mathbf{A})$ Trevigen $^{\circledR}$ XTT assay and (B) Trypan blue exclusion assay.

Notes: $* * P<0.05$. We seeded I0,000 and 50,000 BSG DI0 cells for the XTT and Trypan blue exclusion assays, respectively, before adding varying concentrations of MnPBA488-ANG2 to the cells (studies were performed in triplicate). The cells were coincubated with the nanoparticles for 48 hours at $37^{\circ} \mathrm{C}$ and $5 \% \mathrm{CO}$. The cell viability assays indicated minimal cytotoxicity at nanoparticle concentrations lower than $1.25 \times 10^{-7} \mathrm{mg} / \mathrm{cell}$ after 24 and 48 hours using both assays.

Abbreviations: BSG, brainstem glioma; MnPB, manganese-containing Prussian blue; ANG2, anti-neuron-glial antigen 2.
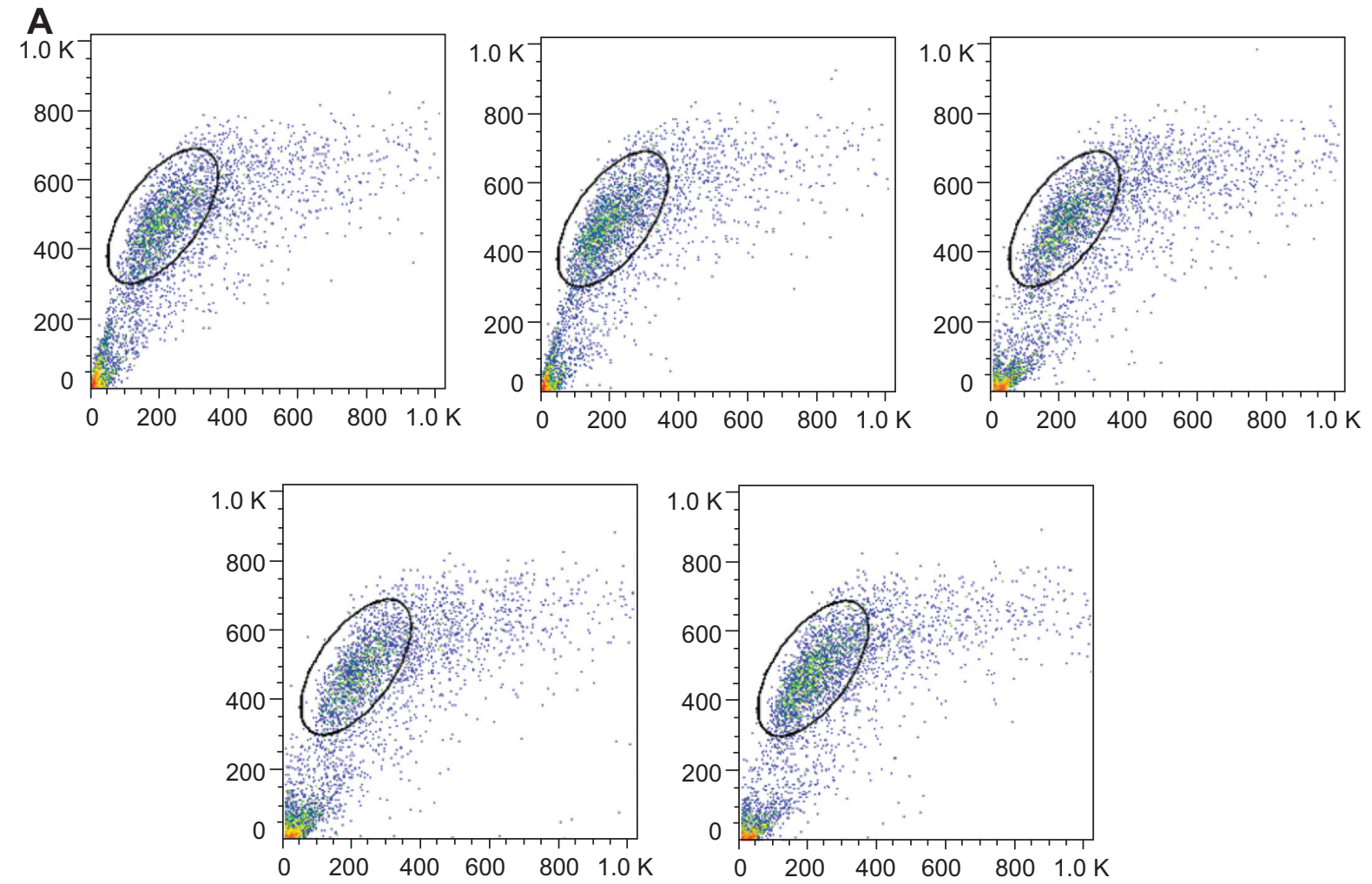

Figure S7 (Continued). 


\section{B}
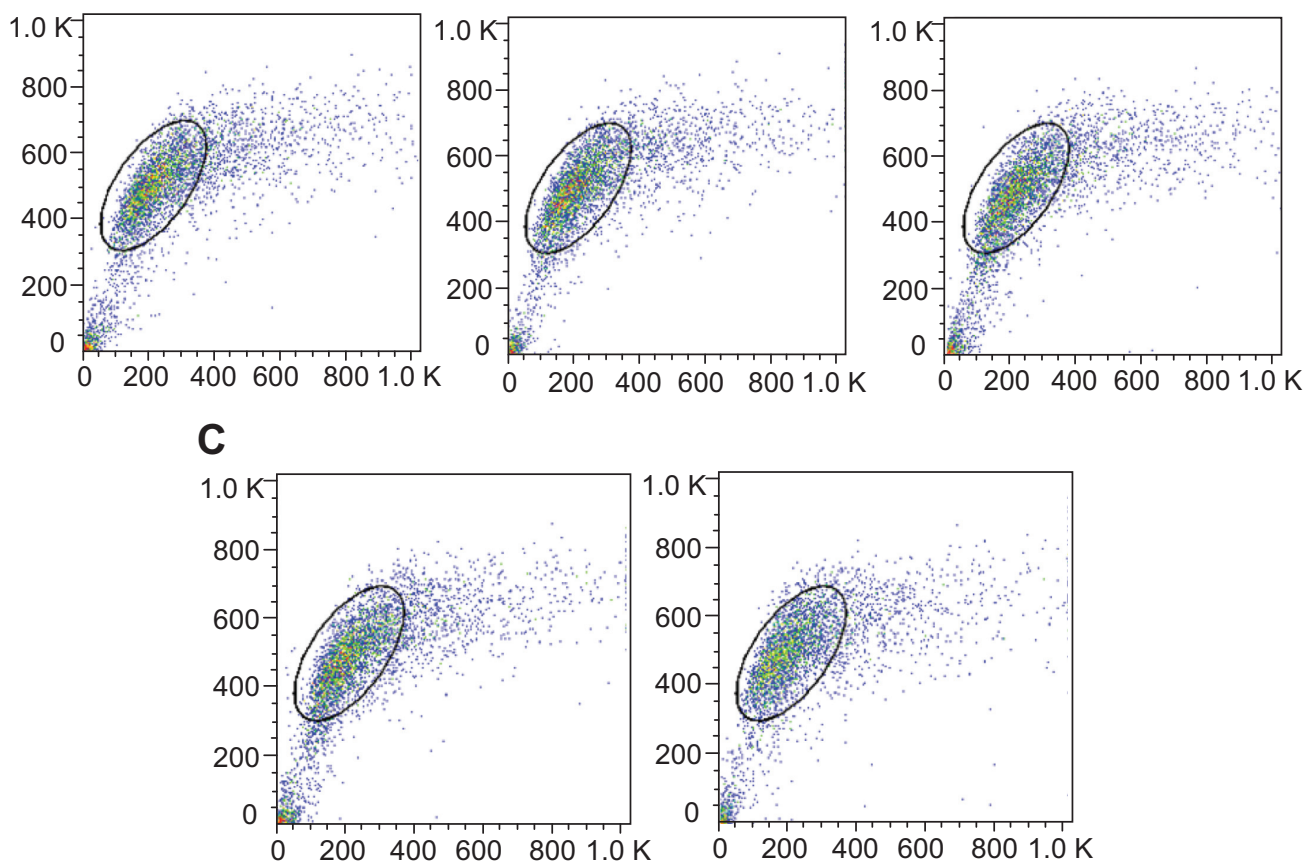

Figure S7 Flow cytometric analysis of nanoparticle specificity for BSG DI0 cells.

Notes: For clarity, the axes legends have been omitted. The side light scattering is displayed on the x-axis (abscissa) and the forward light scattering on the $y$-axis (ordinate). Flow cytometric analysis of the BSG DI0 treated with (A) MnPB-AV488, (B) MnPB-AV488-AbC, and (C) MnPB-A488-ANG2 demonstrated specific targeting of BSG DI0 cells using the targeted MnPB-A488-ANG2 nanoparticles.

Abbreviations: BSG, brainstem glioma; MnPB, manganese-containing Prussian blue; ANG2, anti-neuron-glial antigen 2.

T1

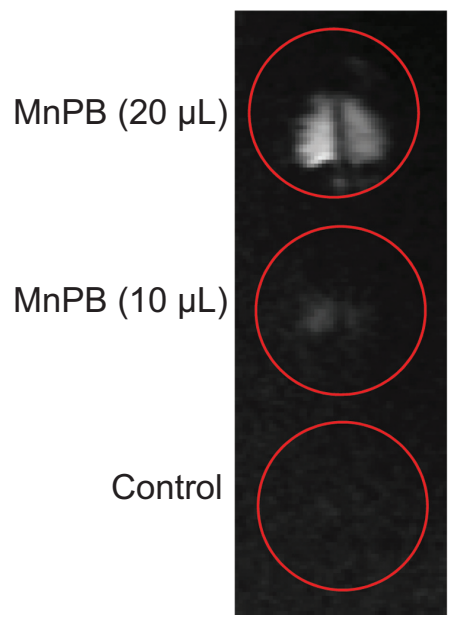

T2

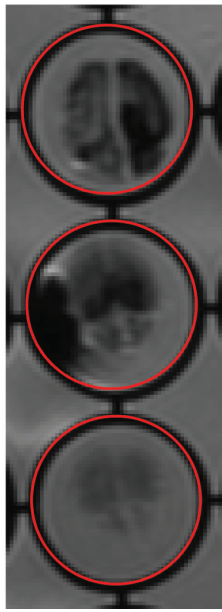

Figure S8 Magnetic resonance images of mouse brains injected ex vivo with two concentrations of MnPB and with saline (control).

Notes: Mouse brains preserved in $10 \%$ formaldehyde in neutral buffer were injected with $10 \mu \mathrm{L}$ or $20 \mu \mathrm{L}$ of a dispersion of $8.5 \mathrm{mg} / \mathrm{mL}$ MnPB or $20 \mu \mathrm{L}$ of saline. The brains were superficially rinsed with Milli-Q water and embedded in $0.5 \%$ agarose prior to imaging. Magnetic resonance images were acquired using clinical GE Healthcare sequences (TI FLAIR: echo train 7; repetition time 2,300 msec; echo time 24.4 msec; matrix size 5 I 2×224, field of view I6 6 I6 cm²; and T2 FRFSE: echo train 2 I; repetition time 3,500 msec; echo time $104 \mathrm{msec}$; matrix size $20 \times 224$; field of view $\left.16 \times 16 \mathrm{~cm}^{2}\right)$.

Abbreviation: MnPB, manganese-containing Prussian blue.

International Journal of Nanomedicine

\section{Publish your work in this journal}

The International Journal of Nanomedicine is an international, peerreviewed journal focusing on the application of nanotechnology in diagnostics, therapeutics, and drug delivery systems throughou the biomedical field. This journal is indexed on PubMed Central, MedLine, CAS, SciSearch ${ }^{\circledR}$, Current Contents ${ }^{\circledR} /$ Clinical Medicine,

\section{Dovepress}

Journal Citation Reports/Science Edition, EMBase, Scopus and the Elsevier Bibliographic databases. The manuscript management system is completely online and includes a very quick and fair peer-review system, which is all easy to use. Visit http://www.dovepress.com/ testimonials.php to read real quotes from published authors.

\footnotetext{
Submit your manuscript here: http://www.dovepress.com/international-journal-of-nanomedicine-journal
} 